\title{
Electrochemical metallization ReRAMs (ECM) - Experiments and modelling: general discussion
}

Ambrosi, Elia ; Bartlett, Philip ; Berg, Sanne; Brivio, Stefano; Burr, Geoffrey ; Deswal, Sweety ; Deuermeier, Jonas ; Haga, Masa-aki ; Kiazadeh, Asal ; Kissling, Gabriela

Total number of authors:

38

Published in:

Faraday Discussions

Link to article, DOI:

10.1039/C8FD90059K

Publication date:

2019

Document Version

Peer reviewed version

Link back to DTU Orbit

Citation (APA):

Ambrosi, E., Bartlett, P., Berg, S., Brivio, S., Burr, G., Deswal, S., Deuermeier, J., Haga, M., Kiazadeh, A., Kissling, G., Kozicki, M., Foroutan-Nejad, C., Gale, E., Gonzalez-Velo, Y., Goossens, A., Goux, L., Hasegawa, T., Hilgenkamp, H., Huang, R., ... Zaffora, A. (2019). Electrochemical metallization ReRAMs (ECM) Experiments and modelling: general discussion. Faraday Discussions, 213, 115-150.

https://doi.org/10.1039/C8FD90059K

\section{General rights}

Copyright and moral rights for the publications made accessible in the public portal are retained by the authors and/or other copyright owners and it is a condition of accessing publications that users recognise and abide by the legal requirements associated with these rights.

- Users may download and print one copy of any publication from the public portal for the purpose of private study or research.

- You may not further distribute the material or use it for any profit-making activity or commercial gain

- You may freely distribute the URL identifying the publication in the public portal 
We have presented the graphical abstract image and text for your article below. This briefly summarises your work, and will be presented with your article online. It will not appear in the print edition.

\section{DISCUSSIONS}

1

\section{Electrochemical metallization ReRAMs (ECM) - Experiments and modelling: general discussion}

Elia Ambrosi, Philip Bartlett, Sanne Berg, Stefano Brivio, Geoffrey Burr, Sweety Deswal, Jonas Deuermeier, Masa-aki Haga, Asal Kiazadeh, Gabriela Kissling, Michael Kozicki, Cina Foroutan-Nejad, Ella Gale, Yago Gonzalez-Velo, Anouk Goossens, Ludovic Goux, Tsuyoshi Hasegawa, Hans Hilgenkamp, Ruomeng Huang, Sherif Ibrahim, Daniele lelmini, Tony Kenyon, Vladimir Kolosov, Yang Li, Sayani Majumdar, Gianluca Milano, Themistoklis Prodromakis, Niloufar Raeishosseini, Vikas Rana, Carlo Ricciardi, Monica Santamaria, Alexander Shluger, Ilia Valov, Rainer Waser, Stanley Williams, Dirk Wouters, Yuchao Yang and Andrea Zaffora

Please check this proof carefully. Our staff will not read it in detail after you have returned it.

Proof corrections must be returned as a single set of corrections, approved by all coauthors. No further corrections can be made after you have submitted your proof corrections as we will publish your article online as soon as possible after they are received.

Please ensure that:

- The spelling and format of all author names and affiliations are checked carefully. Names will be indexed and cited as shown on the proof, so these must be correct.

- Any funding bodies have been acknowledged appropriately.

- All of the editor's queries are answered.

- Any necessary attachments, such as updated images or ESI files, are provided.

Translation errors between word-processor files and typesetting systems can occur so the whole proof needs to be read. Please pay particular attention to: tables; equations; numerical data; figures and graphics; and references.

Please send your corrections preferably as a copy of the proof PDF with electronic notes attached or alternatively as a list of corrections - do not change the text within the PDF file or send a revised manuscript. Corrections at this stage should be minor and not involve extensive changes.

Please return your final corrections, where possible within 48 hours of receipt, by e-mail to: faraday@rsc.org. If you require more time, please notify us by email. 
If any authors have ORCID or ResearcherID details that are not listed below, please provide these with your proof corrections.

Please check that the ORCID and ResearcherID details listed below have been assigned to the correct author. Authors should have their own unique ORCID iD and should not use another researcher's, as errors will delay publication.

Please also update your account on our online manuscript submission system to add your ORCID details, which will then be automatically included in all future submissions. See here for step-by-step instructions and more information on author identifiers.

\begin{tabular}{|c|c|c|c|}
\hline $\begin{array}{l}\text { First (given) } \\
\text { name(s) }\end{array}$ & $\begin{array}{l}\text { Last (family) } \\
\text { name(s) }\end{array}$ & ResearcherID & ORCID \\
\hline Elia & Ambrosi & & \\
\hline Philip & Bartlett & & \\
\hline Sanne & Berg & & \\
\hline Stefano & Brivio & & \\
\hline Geoffrey & Burr & & \\
\hline Sweety & Deswal & & \\
\hline Jonas & Deuermeier & & \\
\hline Masa-aki & Haga & & \\
\hline Asal & Kiazadeh & & \\
\hline Gabriela & Kissling & & \\
\hline Michael & Kozicki & & \\
\hline Cina & Foroutan-Nejad & & \\
\hline Ella & Gale & & \\
\hline Yago & Gonzalez-Velo & & \\
\hline Anouk & Goossens & & \\
\hline Ludovic & Goux & & \\
\hline Tsuyoshi & Hasegawa & & \\
\hline Hans & Hilgenkamp & & \\
\hline Ruomeng & Huang & & \\
\hline Sherif & Ibrahim & & \\
\hline Daniele & lelmini & & \\
\hline Tony & Kenyon & & \\
\hline Vladimir & Kolosov & & \\
\hline Yang & $\mathrm{Li}$ & & \\
\hline Sayani & Majumdar & & \\
\hline Gianluca & Milano & & \\
\hline Themistoklis & Prodromakis & & \\
\hline
\end{tabular}


1

\begin{tabular}{|l|l|l|l|} 
Niloufar & Raeishosseini & & \\
\hline Vikas & Rana & & \\
\hline Carlo & Ricciardi & & \\
\hline Monica & Santamaria & & \\
\hline Alexander & Shluger & & \\
\hline Ilia & Valov & & \\
\hline Rainer & Waser & & \\
\hline Stanley & Williams & & \\
\hline Dirk & Wouters & & \\
\hline Yuchao & Yang & & \\
\hline Andrea & Zaffora & & \\
\hline
\end{tabular}




\section{Queries for the attention of the authors}

Journal: Faraday Discussions

Paper: C8FD90059K

Title: Electrochemical metallization ReRAMs (ECM) - Experiments and modelling: general discussion

For your information: You can cite this article before you receive notification of the page numbers by using the following format: (authors), Faraday Discuss., (year), DOI: 10.1039/ C8FD90059K.

Editor's queries are marked on your proof like this $\mathbf{1}, \mathbf{2}$, etc. and for your convenience line numbers are indicated like this 5, 10, 15,

Please ensure that all queries are answered when returning your proof corrections so that publication of your article is not delayed.

\begin{tabular}{|l|l|l|}
\hline $\begin{array}{l}\text { Query } \\
\text { Reference }\end{array}$ & Query & Remarks \\
\hline & No Queries & \\
\hline
\end{tabular}




\section{DISCUSSIONS}

\section{Electrochemical metallization ReRAMs (ECM) - Experiments and modelling: general discussion}

Elia Ambrosi, Philip Bartlett, Sanne Berg, Stefano Brivio, Geoffrey Burr, Sweety Deswal, Jonas Deuermeier, Masa-aki Haga, Asal Kiazadeh, Gabriela Kissling, Michael Kozicki, Cina Foroutan-Nejad, Ella Gale, Yago Gonzalez-Velo, Anouk Goossens, Ludovic Goux, Tsuyoshi Hasegawa, Hans Hilgenkamp, Ruomeng Huang, Sherif Ibrahim, Daniele lelmini, Tony Kenyon, Vladimir Kolosov, Yang Li, Sayani Majumdar, Gianluca Milano, Themistoklis Prodromakis, Niloufar Raeishosseini, Vikas Rana, Carlo Ricciardi, Monica Santamaria, Alexander Shluger, Ilia Valov, Rainer Waser, Stanley Williams,

Dirk Wouters, Yuchao Yang and Andrea Zaffora

\section{DOI: 10.1039/C8FD90059K}

(1:[1]1) Ella Gale opened discussion of the introductory lecture by Rainer Waser: At the end of your talk you introduced complementary resistance switches, which you used for binary pattern matching. Do you have any insight into how you might use these systems to do computation in the real number space?

Rainer Waser answered: We are currently working on this.

(2:[2]2) Ilia Valov said: From your point of view, at the moment, what are the hottest points of research in memristive cells? What are the main challenges?

Rainer Waser answered: The hot points of research are the physical understanding of the reliability-limiting processes in conjunction with the material's treasure map and, from the application side, the development of a convincing neuromorphic circuit that shows clear benefit compared to standard CMOS solutions

(4:[4]4) Hans Hilgenkamp asked: Most memristor embodiments shown in the papers are based on stacked devices with the current flow perpendicular to the plane. Do all applications necessarily favour this type of configuration, or are there also applications for which an in-plane configuration would be preferred? 
Rainer Waser responded: There are vertical 3D stack concepts, similar to the successful 3D-NAND flash, in which the memristive oxide switches between the stack layer and coverage of the stack. This is a lateral switching.

(5:[5]5) Cina Foroutan-Nejad said: I have a rather naïve question. Do you foresee an opportunity to reduce the variability of memristive devices by downsizing the memristors to the size of a molecule? Is there any attempt that you are aware of to design or make a monomolecular memristor?

Rainer Waser responded: This has been tried for years in the regime of molecular electronics. Unfortunately, these efforts were not successful, presumably it is just not possible to control the contact to a molecule, such as the angle, etc., with sufficient precision.

(6:[6]6) Philip Bartlett asked: You touched on the effects of the interface in your answer to the earlier question. As the memory cells are made smaller in devices any effects of the interfaces between the ReRAM material and the walls of the cells may become more significant. Can you comments on these effects?

Rainer Waser responded: You are right. The effects of the walls of the cells will become more significant with shrinking cell size.

(7:[7]7) Stanley Williams queried: How much do you trust your simulation tools? Most commercial software programs were at least implicitly designed for linear systems near equilibrium. Pushing them into nonlinear systems far from equilibrium exposes them to realms for which they were not designed. How much do you trust the reliability of the simulation results?

Rainer Waser replied: Some of our simulation tools have been developed by ourselves, so that we were able to avoid any limitations. In others, we took care that we were able to use non-linear equations.

(8:[8]8) Themistoklis Prodromakis asked: You presented a variety of memory technologies that each bares its own performance benefits. Do you foresee any opportunities for combining distinct technologies for achieving new functionalities?

Rainer Waser replied: The concept of new functionalities has been presented in the Faraday Discussion articles included in this volume. Please consider the papers on in-memory computing and neuromorphic computing. Some people think about using memristive elements also in sensors.

(9:[9]9) Vladimir Kolosov said: Are there microstructure dependent factors somewhere in the metal oxide switching materials that you have covered in your presentation?

Rainer Waser responded: In my talk I had no time to cover the microstructure aspects. Still, microstructure is very important. There is a huge difference if an oxide material is single crystalline, polycrystalline (e.g. nanocrystalline), or 
amorphous. It is partly covered in our papers, but also the subject of ongoing research.

(10:[10]10) Michael Kozicki opened a general discussion of the paper by Tsuyoshi Hasegawa: It's well known that the conductivity and other properties of silver chalcogenides are highly dependent on what $\delta$ is in the $\mathrm{Ag}_{2+\delta} \mathrm{S}$. What is your $\delta$, and how did you measure it?

Tsuyoshi Hasegawa replied: We did not measure the $\delta$ of our $\mathrm{Ag}_{2+\delta} \mathrm{S}$ nanodot. Although there are several methods for measuring the $\delta$ of a thin $A_{2} g_{2+\delta} S$, they are hard to apply to a nanodot. The $\delta$ of a nanodot should be different from a thin film even when we use the same sulfurization conditions. In the future, we would like to estimate our $\delta$ by comparing with the $\delta$ and resistivity of the bulk values.

(11:[11]11) Stanley Williams said: With respect to the nanowires, how stable are they? Do they want to essentially reform by Ostwald ripening and turn into spheres in order to minimize their surface area?

Tsuyoshi Hasegawa answered: Basically, the reformation of a Ag nanowire into a more stable structure, such as a sphere, should happen as in the question. The volatility of a Ag filament in Ag-based ECM cells seems to be caused by the effect, although a thicker Ag filament stably exists. However, the change in height of a grown Ag nanowire is rarely observed in our experiments using STM. When we grew $\mathrm{Ag}$ nanowires from $\mathrm{Ag}_{2} \mathrm{~S}$ by the e-beam of SEM, we also found that nanowires stably existed for a long time, as long as the e-beam was irradiated. The experimental results suggest that a STM/SEM induced electric field stabilizes a nanowire. The other possibility is that the diameter of the nanowire is thicker than expected. We would like to consider some experiments to confirm what is happening.

(12:[12]12) Ella Gale enquired: In Fig. 3 of your paper (DOI: 10.1039/ C8FD00099A), you compared your data to a theoretical model with a hexagonal symmetry that grew layer by layer, and the experimental results match it very well at the beginning and end of the measured growth process. Did you undertake any modelling of different geometries, for example a slightly slanted layer, to see if it accounts for that discrepancy?

Tsuyoshi Hasegawa answered: We did not try it. When we use a shape that has a wider cross-section in the lower layer, the expected height of the nanowire should become lower than that calculated using a cylindrical shape, seen in Fig. 3 of our paper (DOI: 10.1039/C8FD00099A), and the mismatch becomes larger with growth. However, the mismatch is larger in the intermediate stage in the growth, suggesting other causes. We believe that not all of the $\mathrm{Ag}$ atoms expected to precipitate at each bias did precipitate as such, due to the incomplete inner ionic movement.

(13:[13]13) Dirk Wouters asked: When you are electrochemically removing dopant atoms on one electrode side of the nanodot, shouldn't there be a counter 
reaction at the other electrode side? Similar to the reaction in a conductive bridging RAM and expected in general for electrochemical cells?

Tsuyoshi Hasegawa replied: In the removal or returning of dopant atoms, the following reactions should occur at the surface of the $\mathrm{Ag}_{2} \mathrm{~S}$ interface between the $\mathrm{Ag}_{2} \mathrm{~S}$ and $\mathrm{Ag}$ wire: $\mathrm{Ag}+\mathrm{V}_{\mathrm{I}}^{\mathrm{x}} \rightleftharpoons \mathrm{Ag}_{\mathrm{I}}+\mathrm{e}^{\prime}$

The reaction can occur without any counter reaction at the other electrode side, which is the same as doping into semiconductor materials, or the counter reaction can be described as: ' $+\dot{V_{I}} \rightleftharpoons V_{I}^{x}$

The point of the experiment is that the $\mathrm{Ag}$ atoms that we removed are excess $\mathrm{Ag}$ atoms in the interstitial sites that do not make ionic bonds with sulfur atoms. When $\mathrm{Ag}$ atoms bond to sulfur atoms, a certain counter reaction is expected to occur for keeping the $\mathrm{Ag}_{2} \mathrm{~S}$ matrix, as in the question.

(14:[14]14) Philip Bartlett queried: You use nanosphere lithography to fabricate the $\mathrm{Ag}_{2+\delta} \mathrm{S}$ nanodots. As a result the bases of each nanodot will have an approximately triangular shape and the bottom electrode contact to the Pt has a much larger area than the growing Ag column, is that correct? From the electrochemical perspective this is advantageous because the current density at the larger area contact at the base between the Pt and the nanodot will be much smaller than that at the interface between the growing Ag pillar and the nanodot, such that the Pt nanodot contact acts as a pseudo reference electrode and any changes you implement in the applied potential in the two terminal measurement, such as during the potential (bias) scans, mainly change the potential drop at the silver pillar/nanodot interface.

Tsuyoshi Hasegawa replied: Thank you for the comment. We deposited Ag through a space of nanospheres by EB-deposition. Since the height of the EBdeposited Ag film was $20 \mathrm{~nm}$, which is smaller than the space, the difference in the area size between the top surface and bottom surface is negligible, as far as we observed by STM, AFM, and SEM. Although the sizes of the top surface and bottom surface are almost the same, the current densities at the top surface and the bottom surface are much different, as suggested. We would like to consider the effect when analyzing and doing further experiments.

(15:[15]15) Philip Bartlett said: Following on from my previous question, an obvious approach to ensure a relatively constant reference potential in this type of two electrode experiment would be to use a Ag electrode in place of Pt for the large area back contact.

Tsuyoshi Hasegawa replied: When a Ag electrode is used as a back electrode, $\mathrm{Ag}$ cations are supplied to the $\mathrm{Ag}_{2} \mathrm{~S}$ matrix, keeping the concentration of $\mathrm{Ag}$ cations constant and enabling continuous Ag nanowire growth. We reported such experimental results in our previous work. ${ }^{1}$ In order to achieve atom by atom precipitation as a function of bias, we could not use $\mathrm{Ag}$ as a back electrode.

1 K. Terabe, T. Nakayama, T. Hasegawa and M. Aono, J. Appl. Phys., 2002, 91, 10110. 
(16:[16]16) Ilia Valov remarked: The electronic conductivity of the low temperature form of $\mathrm{Ag}_{2} \mathrm{~S}$ is very sensitive to doping with $\mathrm{Ag}$ atoms. $\mathrm{Ag}_{2} \mathrm{~S}$ is a semiconductor with $\sim 3 \mathrm{eV}$ bandgap. When $\mathrm{Ag}$ atoms dissolve in the sulfide matrix they dissociate to $\mathrm{Ag}^{+}$ions and electrons. Whereas the ionic conductivity is not influenced by this doping, the electronic conductivity is significantly increased. Therefore, when one removes $\mathrm{Ag}$ atoms from $\mathrm{Ag}_{2} \mathrm{~S}$ nanodots (containing only a limited number of dopant atoms) we observe a discrete and significant change of the electronic conductivity.

(17:[17]17) Gabriela Kissling asked: How reversible is the formation of the silver nanowires? Do the silver ions get reabsorbed and evenly redistributed in the $\mathrm{Ag}_{2+\delta} \mathrm{S}$ nanoparticle when the nanowire shrinks or does the silver form a kind of core-shell structure on the outside of the nanoparticle?

Tsuyoshi Hasegawa answered: Nanowire growth and shrinkage is repeatable, as we reported previously. ${ }^{1}$ Since we remove the $\mathrm{Ag}$ atoms that only existed in the interstitial sites, they easily move back to the interstitial sites in the $\mathrm{Ag}_{2} \mathrm{~S}$ matrix. Removing the $\mathrm{Ag}$ atoms that bond to sulfur atoms breaks the $\mathrm{Ag}_{2} \mathrm{~S}$ matrix, making it impossible for the $\mathrm{Ag}$ atoms to return.

1 K. Terabe, T. Nakayama, T. Hasegawa and M. Aono, J. Appl. Phys., 2002, 91, 10110.

(18:[18]18) Geoffrey Burr asked: Have you considered using your technique for nano-stereo-lithography? That is, you would use the STM tip to pull some metal atoms out into a nanowire, then hold the potential while you deposited, very gently, a small number of dielectric atoms in the vicinity of the wire. This would require the formation of the filament to be stable enough that you can hold it in place while you "encase" it in non-conducting atoms and/or molecules.

Tsuyoshi Hasegawa replied: No, we have not considered using our technique for nano-stereo-lithography. This is because the chemical reaction that precipitates the $\mathrm{Ag}$ atoms occurs at the interface between a grown nanowire and a solid electrolyte, such as $\mathrm{Ag}_{2} \mathrm{~S}$. This means that our technique cannot grow another branch from a nanowire surface.

(52:[19]19) Asal Kiazadeh remarked: Thanks for presenting your work at the Faraday Discussion. Since the nanowire is not stable enough and basically the retention time is low, I would like to know have you ever tried some other experiments, such as pulse train measurement by applying different amplitudes/ frequencies, in order to study the plasticity of Ag dynamics? I mean, could you use the measurement set-up shown in Fig. 2 and 3 of the work by Kim and Lee ${ }^{1}$ as a follow-up study?

Tsuyoshi Hasegawa responded: Ag nanowire growth and its stability, such as under pulse train, is partly reported in our previous work, ${ }^{1}$ although the $\mathrm{Ag}$ nanowires grow in a molecular layer in the study. Regarding the experiment using STM in vacuum, we reported this previously. ${ }^{2}$ Regarding the technique used, it 
was used for the first time by ourselves in this article (DOI: 10.1039/C8FD00099A). In addition to this paper, we have also reported the STM and LTP characteristics previously. $^{3-6}$

1 C. Arima, A. Suzuki, A. Kassai, T. Tsuruoka and T. Hasegawa, J. Appl. Phys., 2018, 124, 152114.

2 T. Ohno, T. Hasegawa, T. Tsuruoka, K. Terabe, J. K. Gimzewski and M. Aono, Nature Mater., 2011, 10, 591-595.

3 T. Ohno, T. Hasegawa, A. Nayak, T. Tsuruoka, J. K. Gimzewski and M. Aono, Appl. Phys. Lett., 2011, 99, 203108.

4 R. Yang, K. Terabe, G. Liu, T. Tsuruoka, T. Hasegawa, J. K. Gimzewski and M. Aono, ACS Nano, 2012, 6, 9515-9521.

5 A. Nayak, T. Ohno, T. Tsuruoka, K. Terabe, T. Hasegawa, J. K. Gimzewski and M. Aono, Adv. Func. Mater., 2012, 22, 3606.

6 R. Yang, K. Terabe, T. Yao, T. Tsuruoka, T. Hasegawa, J. K. Gimzewski and M. Aono, Nanotechnology, 2013, 24, 384003.

(19:[20]20) Stanley Williams opened a general discussion of the paper by Yuchao Yang: Have you looked for the threshold voltages in your anodic and cathodic reactions at the oxide/electrode interfaces? Is there a characteristic voltage or voltages below which you don't see one or the other? Can you control which reaction occurs?

Yuchao Yang replied: Thanks for raising this interesting question. Indeed, the occurrence of both anodic and cathodic reactions requires certain threshold voltages to be reached. We have performed a voltage dependent study on the anodic and cathodic reactions for the same $\mathrm{Pt} / \mathrm{HfO}_{2} / \mathrm{Pt}$ system. It can be observed that the reactions start to take place above $8 \mathrm{~V}$. However, there is difficulty in completely decoupling them since the anodic and cathodic processes happen simultaneously in order to maintain overall charge neutrality. As a result, we always saw the anodic and cathodic processes happening in parallel, otherwise no reactions occurred at low voltages. We expect that in the case of multiple anodic or cathodic processes, it could be possible to control their occurrence using the applied voltage.

(20:[21]21) Masa-aki Haga asked: You provide the $I-V$ profile of the $\mathrm{Pt} / \mathrm{HfO}_{2} / \mathrm{Pt}$ devices in Fig. $1 l$ of your article (DOI: 10.1039/C8FD00113H) and you said that "a cathodic reaction must take place simultaneously with the anodic reaction". How many volts are needed to cause such reactions? I am wondering why these reactions did not affect the current flow or $I-V$ characteristics since holes were created during the potential scanning. I saw the reverse current was undulating in Fig $4 l$ of your article. I expected similar undulation of the current when the holes were generated during the potential scanning.

Yuchao Yang replied: In Fig. 2b-e of our article (DOI: 10.1039/C8FD00113H), we have performed a voltage dependent study on the anodic and cathodic reactions for the same $\mathrm{Pt} / \mathrm{HfO}_{2} / \mathrm{Pt}$ system. It can be observed that the reactions start to take place above $8 \mathrm{~V}$. The undulation of the current may not always be observed, since the conductivities in the $\mathrm{HfO}_{2}$ and $\mathrm{TaO}_{\mathrm{x}}$ based memristors are mainly provided by the localized conducting filaments instead of being a homogeneous effect. As a result, it can be found that the formation of holes/damage to the electrodes did not introduce current undulation in Fig. 1, 3, 5, and 6 of our article. 
The only exception is the current undulating in the off state shown in Fig. $4 l$, which could be related to the occurrence of the redox reactions. The conductance of the device is fully dominated by the electronic current offered by the conducting filament once the device is switched to on state, and hence such undulation as a result of the redox processes cannot be observed.

(21:[22]22) Masa-aki Haga asked: How thin are the films on the $\mathrm{Pt} / \mathrm{HfO}_{2} / \mathrm{Pt}$ devices?

Yuchao Yang responded: The thickness of the $\mathrm{HfO}_{2}$ films in the $\mathrm{Pt} / \mathrm{HfO}_{2} / \mathrm{Pt}$ devices is $30 \mathrm{~nm}$.

(22:[23]23) Rainer Waser said: I wonder if you have changed the humidity in order to see if you can control the hydrogen evolution and suppress it using a dry atmosphere?

Yuchao Yang answered: This is a great suggestion. The experiments in this work were performed in air where the humidity level could not be controlled, but it is a very interesting suggestion to vary the level of moisture and examine the cathodic reactions.

(23:[24]24) Alexander Shluger remarked: What is the evidence that the water adsorbed on the hafnia surface does not dissociate by itself through adsorption and requires the reaction with the electrons in order to provide the $\mathrm{OH}^{-}$reaction, which is reaction 2 in the article (DOI: 10.1039/C8FD00113H)? Water tends to dissociate at surfaces consisting of $\mathrm{SiO}_{2}, \mathrm{TiO}_{2}$, and some other oxides.

Yuchao Yang answered: The necessity for the reaction of water with electrons originates from the general requirement for charge neutrality, since the anodic reactions described by eqn 1 of the article (DOI: 10.1039/C8FD00113H) will release electrons. This makes the generation of the $\mathrm{OH}^{-}$ions described by eqn 2 in the article a more probable reaction at the cathode interface.

(24:[25]25) Philip Bartlett asked: Just to clarify, are you talking about the dissociation of water into $\mathrm{H}^{+}$and $\mathrm{OH}^{-}$on the oxide surface or a redox process that generates hydrogen, which is reaction 2 in the paper (DOI: 10.1039/C8FD00113H), or are you referring to one of the other reactions in the paper, for example reaction 3 or 4 ?

Alexander Shluger replied: My question was, what is the evidence that the water adsorbed on the hafnia surface does not dissociate by itself through adsorption and requires the reaction with the electrons in order to provide $\mathrm{OH}^{-}$? Water tends to dissociate on the surfaces of $\mathrm{SiO}_{2}, \mathrm{TiO}_{2}$, and some other oxides. There have been suggestions regarding water dissociation on the surfaces of $\mathrm{HfO}_{2}$ in the studies by Navrotsky et al. ${ }^{1}$ and Driemeier and Gusev. ${ }^{2}$ The nature of reaction 2 in the paper (DOI: 10.1039/C8FD00113H) is unclear. Such reaction takes place during the radiolysis of water, but how it may take place as "the cathodic reaction was suggested to be the reduction of moisture absorbed in the memristive oxides" is unclear. If water dissociates due to the interaction with the 
surface, then $\mathrm{H}$ can be formed when the injected electrons interact with $\mathrm{H}^{+}$at a certain Fermi level position. ${ }^{3}$ You see damage caused, but you do not know whether it is caused by hydrogen or not. Do you have any evidence that hydrogen produces this damage?

1 G. Sharma, S. V. Ushakov, H. Liu, R. H. R. Castro and A. Navrotsky, J. Phys. Chem. C, 2017, 121, 10392-10397.

2 C. Driemeier and E. P. Gusev, Appl. Phys. Lett., 2006, 88, 201901.

3 M. Kaviani, V. V. Afanas'ev and A. L. Shluger, Phys. Rev. B, 2017, 95, 075117.

Yuchao Yang responded: The assumption of hydrogen evolution is based on previous experimental evidence in the literature showing that moisture is indispensable for cathodic reactions and forming was not possible without the presence of moisture, such as in the papers by Lübben et al. ${ }^{1,2}$ Since the oxidation of the oxygen ions at the anodic interface will create oxygen vacancies, a counter electrode reaction generating anions is required to keep the charge neutrality, thus suggesting the reduction of moisture to $\mathrm{OH}^{-}$ions and accompanying hydrogen gas. Certainly, this is not direct evidence and it would be nice to directly analyze the chemistry of the gas products from the cathodic interface.

1 M. Lübben, S. Wiefels, R. Waser and I. Valov, Adv. Electron. Mater., 2018, 4, 1700458.

2 M. Lübben, P. Karakolis, V. Ioannou-Sougleridis, P. Normand, P. Dimitrakis and I. Valov, Adv. Mater., 2015, 27, 6202-6207.

(26:[27]27) Tony Kenyon asked: Have you tried to detect what gas is emitted during the forming and switching events? Related to that, what do you think the contribution is of local heating in producing the damage that you see?

Yuchao Yang answered: At this moment we do not have experimental means that can directly analyze the chemistry of the gas products during the forming and switching events. The contribution of local Joule heating is indeed present. In fact, the evolution of gas products from the anodic and cathodic interfaces is not expected to cause structural damage to the bottom electrode, which sits underneath both interfaces. However, such damage was indeed observed in Fig. 1-6 of our article (DOI: 10.1039/C8FD00113H), which could be induced by the local heating effects. By lowering the current compliance and thus reducing the Joule heating, the damage to the bottom electrodes could be effectively prevented, as shown in Fig. $2 \mathrm{f}$ and $4 \mathrm{~m}$ of our paper, further supporting this hypothesis. In addition, the periphery of the damaged area usually exhibits morphology that indicates a preceding melting process, e.g. in Fig. 1g, once again suggesting the role of Joule heating in producing the structural damage.

(27:[28]28) Carlo Ricciardi said: My question is about Fig. 7 of your article (DOI: $10.1039 / \mathrm{C} 8 \mathrm{FD} 00113 \mathrm{H}$ ). Can you specify if the $\mathrm{ZnO}$ film is amorphous or nano/ polycrystalline? If it is the latter case, do you see any effect from the microstructure like localization of the conductive filament in the grain boundaries?

Yuchao Yang responded: Thanks for raising this interesting point. The $\mathrm{ZnO}$ film in our device is nanocrystalline, as can be found from the topography of the films (Fig. 7a, d, g, and j). Although this work did not focus on the spatial correlation of the filament locations and grain boundaries, previous AFM and 
CAFM studies on the $\mathrm{Cu} / \mathrm{ZnO} / \mathrm{Pt}$ devices have shown that $\mathrm{Cu}$ filaments tend to form at the $\mathrm{ZnO}$ grain boundaries, as seen in the work by Zhuge et al., ${ }^{1}$ demonstrating the validity of such correlation.

1 F. Zhuge, S. Peng, C. He, X. Zhu, X. Chen, Y. Liu and R. Li, Nanotechnology, 2011, 22, 275204 .

(29:[29]29) Ilia Valov commented: I agree with you that charging of the thin films can be a source of instability. However, if you look at the images, there is development of gas at both electrodes. It's obvious that gas is developing. If there's damage then it's gas. It cannot be something else. It is an electrochemical reaction that takes place at both electrodes upon polarization. We have also observed $\mathrm{H}_{2}$ and $\mathrm{O}_{2}$ evolution during polarization in our work. ${ }^{1,2}$

1 M. Lübben, S. Wiefels, R. Waser and I. Valov, Adv. Electron. Mater., 2018, 4, 1700458. 2 I. Valov and T. Tsuruoka, Phys. D Appl. Phys., 2018, 51, 413001.

Alexander Shluger answered: The statement in the paper (DOI: 10.1039/ C8FD00113H) that a cathodic reaction must take place simultaneously with the anodic reaction in both the ECM and VCM cells, in order to maintain charge neutrality, is not applicable to the open systems considered here. The charging of oxides in electronic devices is well known in the microelectronics community and leads to oxide degradation. The results provided in Fig. 1m of your paper (DOI: 10.1039/C8FD00113H) show delamination of the Pt electrode but no $\mathrm{H}_{2}$ gas is detected. Although some hydrogen gas formation may take place at the $\mathrm{HfO}_{2}$ surface, see the work by Driemeier and Gusev ${ }^{1}$ and Navrotsky et al. ${ }^{2}$ this paper does not provide proof of that happening and delamination can be caused, for example, by interfacial stress due to the structural changes caused by the significant loss of oxygen.

1 C. Driemeier and E. P. Gusev, Appl. Phys. Lett., 2006, 88, 201901.

2 G. Sharma, S. V. Ushakov, H. Li, R. H. R. Castro and A. Navrotsky, J. Phys. Chem. C, 2017, 121, 10392-10397.

(30:[30]30) Daniele Ielmini remarked: The forming operation in your devices induces local bubbling and eruption. Do the samples still display resistance switching characteristics with set/reset operation after forming? Can you comment on the impact of scaling on the forming/set/reset behavior in your material system?

Yuchao Yang answered: The devices can be successfully set and reset after going through the gas eruptions and forming process. Similar phenomena have been observed in many previous studies ${ }^{1,2}$ where the devices all showed normal set/reset operations after gas formation, indicating the generality of such behavior in oxide based memristors. As the device is scaled down, the number of filaments and the extent of interfacial reactions might be reduced to a certain extent. However, since the resistive switching is filamentary in such systems, instead of a homogeneous effect, the forming/set/reset behaviors are expected to be similar in general. 
1 J. J. Yang, F. Miao, M. D. Pickett, D. A. A. Ohlberg, D. R. Stewart, C. N. Lau and R. S. Williams, Nanotechnology, 2009, 20, 215201.

2 D. Kwon, K. M. Kim, J. H. Jang, J. N. Jeon, M. H. Lee, G. H. Kim, X. Li, G. Park, B. Lee, S. Han, M. Kim and C. S. Hwang, Nat. Nanotechnol., 2010, 5, 148-153.

(44:[31]31) Monica Santamaria asked: Since you performed the voltage-current experiments under air exposure, did you consider the $\mathrm{O}_{2}$ reduction as an additional faradaic process that can occur at the cathodic interface? The standard equilibrium potentials of the $\mathrm{O}_{2} / \mathrm{H}_{2} \mathrm{O}$ or $\mathrm{O}_{2} / \mathrm{OH}^{-}$equilibria are both more positive than the standard equilibrium potential of the $\mathrm{H}_{2} \mathrm{O} / \mathrm{H}_{2}$ equilibria, thus they are thermodynamically more favourable that the latter. This process can account for the lower volume of evolved gas at the cathode side.

Yuchao Yang answered: Thanks for the suggestion. We fully agree that the reduction of oxygen might also be responsible for the lower volume of gas evolved from the cathodic interface that we observed. The detailed cathodic process will be in the scope of future studies.

(45:[32]32) Monica Santamaria remarked: If you perform the $I-V$ experiments under vacuum, you simultaneously reduce the moisture and oxygen concentrations. Did you check if the forming occurs under vacuum conditions?

Yuchao Yang answered: All the experiments were performed in ambient air in the present case. However, previous studies on similar material systems using vacuum conditions have revealed that the forming process is not possible when the device was placed in vacuum and heated to remove adsorbed moisture. ${ }^{1}$

1 M. Lübben, S. Wiefels, R. Waser and I. Valov, Adv. Electron. Mater., 2018, 4, 1700458.

(46:[33]33) Ilia Valov commented: If you use moisture free oxygen and oxygen free moisturized $\mathrm{N}_{2}$ it can be possible to distinguish between these effects.

Yuchao Yang answered: Thanks for the suggestion. For the present study, all experiments were performed in air where the humidity level could not be controlled, but it will be a very interesting point to decouple the roles of oxygen and moisture in interfacial reactions based on this approach.

(47:[34]34) Jonas Deuermeier asked: Have you observed the bubbles occurring at the edges or rather in the centre of your devices? If so, was there a change when you used different polarities for switching?

Yuchao Yang answered: As can be found from Fig. 1-6 of our paper (DOI: 10.1039/C8FD00113H), in memristive systems with varied oxide, electrode combination, applied voltage, and current compliance, the bubbles are always emitted from the centre of the devices without showing preference to the edge of the electrodes. We have also adopted reversed voltage polarity in Fig. 4 and 5 of the article and the sites of bubble evolution showed generally the same results. However, by reversing the voltage polarity, it was found that the anodic reactions (now the bottom electrode in Fig. 4 and 5 of our paper) always resulted in more pronounced damage to the corresponding electrode, hence indicating the 
possible existence of additional reduction processes that do not produce gaseous products.

(48:[35]35) Ella Gale commented: I did a similar study with much larger memristors and I saw bubbles start to form at the edge of the cross bars area, see Fig. 9 in my previous work in Materials Chemistry and Physics. ${ }^{1}$ I thought this might be due to the increase in strain in the semiconductor and the top electrode at the edge of the cross-bar. It might be interesting to investigate that.

1 E. Gale, D. Pearson, S. Kitson, A. Adamatzky and D. L. Costello, Mater. Chem. Phys., 2015, 162, 20-30.

Yuchao Yang replied: Thanks for pointing out that interesting work. Yes, it is possible that the increased strain at the edges could contribute to the generation of bubbles, leading to gas evolution at the edges. Moreover, the electric field at the electrode edges is usually enhanced and might also play a role. However, it could still depend on the detailed conditions in the systems studied. As can be found from Fig. 1-6 in the article (DOI: 10.1039/C8FD00113H), in the present memristive systems with varied oxide, electrode combination, applied voltage, and current compliance, the sites of bubble generation did not exhibit any specific preference to the edges but were distributed randomly on the top of the electrodes.

(49:[36]36) Anouk Goossens said: In Fig. 3-6 of your article (DOI: 10.1039/ C8FD00113H), you show some global current-voltage measurements conducted over the entire electrode area. Have you also conducted any local current-voltage measurements in order to determine how the structural damage to the electrodes affects conductivity? How large is the difference in conductance between the areas with significant damage and the pristine regions?

Yuchao Yang responded: We did not measure the localized conductance in the present study, but previous studies showing similar structural damage have revealed enhanced conductivity (by a factor of $\sim 4$ ) in the areas showing structural damage compared with the pristine region, ${ }^{1}$ in agreement with the generation of oxygen vacancies in the reactions. We expect similar behavior holds in the present case.

1 K. Skaja, C. Bäumer, O. Peters, S. Menzel, M. Moors, H. Du, M. Bornhöfft, C. Schmitz, V. Feyer, C. Jia, C. M. Schneider, J. Mayer, R. Waser and R. Dittmann, Adv. Funct. Mater., 2015, 25, 7154-7162.

(50:[37]37) Ruomeng Huang asked: According to Fig.1a of your paper (DOI: $10.1039 / \mathrm{C} 8 \mathrm{FD} 00113 \mathrm{H})$, the probe for the $I-V$ measurements has been placed at the edge of each device. Is there any particular reason for this? Have you tried to place the probe in the centre of the device to see the impact the probe position might have on the bubble forming?

Yuchao Yang answered: The probe was placed at the edge of the device in order to minimize its influence on the observation of gas evolution and electrode damage. Also, qualitatively the same results have been observed when the probe 
was placed in the centre of the electrode, as long as the loading force was kept at a low level.

(51:[38]38) Ruomeng Huang commented: The formation of bubbles means damage to the device. Did you observe this kind of damage in the subsequent SET and RESET processes or is it exclusive to the electro-forming process? In addition, you have demonstrated the damage can be mitigated by forming the device in an opposite polarity. In terms of the device performance, did you observe any difference in the performance for these two devices when using opposite forming polarities?

Yuchao Yang responded: The electrode damage was predominantly introduced in the forming process, while the subsequent set and reset processes are mainly caused by the redistribution of the ionic species introduced during forming. As a matter of fact, the redox processes were the same when the voltage polarity was reversed and only the positions for the anodic and cathodic reactions were switched, for example in Fig. 4 and 5 of the article (DOI: 10.1039/C8FD00113H). However, by reversing the voltage polarity, it was observed that the anodic reactions (now the bottom electrode) always resulted in more pronounced damage to the corresponding electrode, hence indicating the possible existence of additional reduction processes that do not produce gaseous products.

(53:[39]39) Alexander Shluger said: I have a general comment about the gas emission from these devices under high bias. This effect has already been observed in the sixties and seventies. ${ }^{1}$ Whether bubbles are observed on the top of the top electrode or on the sides depends on the way that the electrode is deposited. Sputter deposition, often used in experiments, creates columnar structures with grain boundaries. ${ }^{2}$ Oxygen can percolate through these grain boundaries creating bubbles on the top. If the electrodes have stronger adhesion to the oxide and less grain boundaries, the pressure of the oxygen gas can create delamination and bubbles on the sides of the electrode.

1 G. Dearnaley, A. M. Stoneham and D. V. Morgan, Rep. Prog. Phys., 1970, 33, 1129. Fearn, D. McPhail, M. Bosman, A. L. Shluger and A. J. Kenyon, Adv. Mater., 2016, 28, 7486.

Yuchao Yang responded: Thanks for sharing these important and relevant comments. The dependence of the gas emission sites on the microstructure and adhesion of the electrodes is highly plausible to us. In the present study all the electrodes were deposited by electron beam evaporation, which could give rise to similar microstructures to those in electrodes deposited by sputtering. It was found that the gas bubbles always emitted randomly from the top of the electrodes in our case, seen in Fig. 1-6 of our paper (DOI: 10.1039/C8FD00113H), and this might be related to the percolation of the gas molecules through the grain boundaries of the electrodes.

(54:[40]40) Michael Kozicki remarked: You have a pretty well defined mechanical system. Coming back to the gas evolution at the metal electrode, you know the material properties of the metal, shear strength, thickness, other 
dimensions, etc. Do you have any idea of what the gas pressure is that's causing the electrode deformation and if that could be used to give clues about its evolution?

Yuchao Yang replied: Thanks for raising this point. It will be very interesting to estimate the gas pressure that leads to the observed electrode deformations and thus the extent of the preceding redox reactions. Nevertheless, construction of such a model is complicated by other factors, such as the varied local temperature during resistive switching, which evolves during the switching process and could significantly change the mechanical properties of the electrodes, making them different from that at room temperature. Such local temperature during resistive switching is not well documented yet.

(55:[41]41) Philip Bartlett said: In the paper (DOI: 10.1039/C8FD00113H), you used both Pt and Pd electrodes in different devices. Hydrogen evolution on Pd is different from Pt because the evolved hydrogen can dissolve into the Pd to make the alpha or beta phase palladium hydride. Did you see any evidence of different behavior for the Pt and Pd?

Yuchao Yang responded: Both the electrical behaviors and structural damage in the devices with Pt and Pd electrodes were very similar, as shown in Fig. 1-5 of the paper (DOI: 10.1039/C8FD00113H). The suggested hydrogen dissolution in Pd could play a role but did not appear to have a pronounced effect, which might be due to the fact that the reaction in eqn 2 of the paper was not the only cathodic process in the devices. Instead, our results indicated the possible existence of additional reduction processes that do not produce gaseous products, e.g. the reduction of Hf or Ta cations to their lower valence states.

(800:[42]42) Andrea Zaffora communicated: You mentioned that the extent of the redox processes in the $\mathrm{Pt} / \mathrm{HfO}_{2} / \mathrm{Pt}$ device can be manipulated by the amplitude of the forming voltage. How can you change the forming voltage in this device without changing the oxide thickness or stoichiometry? It is clearly shown in Fig. 1a of the paper (DOI: 10.1039/C8FD00113H) that, for the device you mentioned, the forming voltage is higher than $12 \mathrm{~V}$.

Yuchao Yang communicated in reply: The varied forming voltage in the present case refers to the amplitude of the voltage that was adopted during the forming process instead of the forming threshold, which was changed from 8 to $14 \mathrm{~V}$ with a fixed current compliance. One can see that the extent of the redox processes and electrode damage gradually increased as the voltage amplitude was increased. It should be pointed out that such redox processes and electrode damage already occurred even before the forming threshold was reached (e.g. at 8 V), as shown in Fig. 2 of the paper, indicating that the forming process is actually a consequence of such redox processes.

(801:[43]43) Gianluca Milano communicated: In your work, you have investigated interfacial redox processes in ECM systems by considering $\mathrm{Cu} / \mathrm{ZnO}$-based ECM devices. However, $\mathrm{ZnO}$ is well-known to be able to also switch in VCM configuration with an oxygen vacancy related mechanism. Indeed, as correctly 
stated by the author, the formation of oxygen vacancies and oxygen ions cannot be excluded in $\mathrm{ZnO}$. For example, as reported in previous work, ${ }^{1} \mathrm{Cu} / \mathrm{ZnO}$-based devices can be switched with a VCM mechanism by forming a conductive path with a positive polarity applied to the electrochemically inert electrode, instead of to the electrochemically active $\mathrm{Cu}$ electrode. These observations reveal that, besides the electrochemical reactions reported in eqn 6 and eqn 7 of your manuscript (DOI: 10.1039/C8FD00113H), the electrochemical reactions observed in VCM systems, such as eqn 1 in your manuscript, can be still present in $\mathrm{Cu} / \mathrm{ZnO}$ based devices. Did you observe any effect related to the VCM electrochemical reactions also in the $\mathrm{Cu} / \mathrm{ZnO}$ ECM systems? Can you exclude an interdependence of both mechanisms in $\mathrm{Cu} / \mathrm{ZnO}$ devices?

1 G. Milano, S. Porro, M. Y. Ali, K. Bejtka, S. Bianco, F. Beccaria, A. Chiolerio, C. F. Pirri and C. Ricciardi, J. Phys. Chem. C, 2017, 122, 866-874.

Yuchao Yang communicated in reply: Thanks for raising a good point. We fully agree that the electrochemical reactions related to the valence change mechanism in $\mathrm{ZnO}$ can be present in the $\mathrm{Cu} / \mathrm{ZnO}$ system. This issue was mitigated in the present study since the $\mathrm{Cu}$ electrode was adopted and positively biased. In this case, it is expected that the oxidation of the $\mathrm{Cu}$ metal atoms to $\mathrm{Cu}$ ions should be the dominant anodic process because the oxidation of the oxygen ions, described by eqn 1 in the manuscript (DOI: 10.1039/C8FD00113H), requires a much higher voltage. Indeed, the experimental results also revealed the migration of the $\mathrm{Cu}$ ions and subsequent nucleation at the cathodic interface, seen in Fig. 7 of the article, without showing clear evidence involving the VCM mechanism in the experimental conditions used in this work.

(31:[44]44) Alexander Shluger opened the discussion of the paper by Yago Gonzalez-Velo: How large is the effect of radiation with respect to the variability in the characteristics of your devices?

Yago Gonzalez-Velo responded: Variability is always a concern with ReRAM devices. In our case, we are careful to mention effects when an observed variation of an electrical parameter is large enough to be representative. Additionally, with the chalcogenide based PMCs (ReRAM) cells that we are able to make, we do find that the electrical parameters exhibit good repeatability and also that such a material system has been used for a long time even in commercially released ReRAM standalone memory. We previously performed total dose exposure of such commercial memory and limited effects were observed. ${ }^{1}$

1 Y. Gonzalez-Velo, H. J. Barnaby, M. N. Kozicki, C. Gopalan and K. Holbert, IEEE Electron Device Lett., 2014, 35, 205-207

(33:[46]46) Stanley Williams asked: Have you tried to make any estimates for the integrated energy deposited into your films by the radiation exposure that you deliver? I recommend that you calculate the deposited energy in terms of a more familiar quantity such as eV or whatever per atom within your film. This may be a useful exercise to go through to understand how the energy deposited into the system compares to the thermal energy. 
Yago Gonzalez-Velo answered: The total dose provided is given as $\operatorname{rad}\left(\mathrm{Ge}_{30}\right.$ $\left.\mathrm{Se}_{70}\right)$ and Gray $(1 \mathrm{~Gy}=100 \mathrm{rad})$, which is the standard unit. They represent the amount of energy absorbed per unit mass. Consequently, the calculation of the energy deposited in the volume of the active ReRAM via is possible and can be done by the following: Total Dose $\times$ mass. The via is about $10 \mu \mathrm{m}$ in diameter and $60 \mathrm{~nm}$ in thickness, so its mass is close to $23.510 \mathrm{E}^{-12} \mathrm{~kg}$ consisting of about 5 $\mathrm{gcm}^{-3}$. This leads to an energy absorbed close to $1.47 \mathrm{E}^{12} \mathrm{eVMrad}^{-1}$ of the total ionising dose (TID), so in our case 1.06 Mrad leads to Eabsorbed(TID_1)=1.55 $10 \mathrm{E}^{12} \mathrm{eV}$ and $2.38 \mathrm{Mrad}$ leads to Eabsorbed(TID_2) $=3.4910 \mathrm{E}^{12} \mathrm{eV}$.

(35:[48]48) Ella Gale queried: In Fig. 5 of your paper (DOI: 10.1039/ C8FD00125A), you conclude that the radiation-dosage effect on annealed devices was monotonic (unlike the non-annealed devices), but why did you do only two different quantities of radiation? I do not think that you can conclude the dosage response is monotonic with only two dosages values.

Yago Gonzalez-Velo replied: In Fig. 5 of the article (DOI: 10.1039/C8FD00125A), you can observe the behavior as a function of TID for three points at increasing total dose (0M rad, $1.06 \mathrm{Mrad}$, and 2.38 Mrad). As a consequence, we can say that experimentally, according to the results obtained and available, the response is monotonic, whereas it is not in the case of samples that were not annealed. The choice of 2 dose steps is based on setting up the experiment and being able to conduct the needed characterization in a timely manner, including the electrical testing of several devices repeatedly and XRD without having samples out of the irradiation chamber for long periods of time to minimize the relaxation effects

(36:[49]49) Ella Gale asked: Following on from my previous question, with the data that you have, can you tell me how long these devices would last in space?

Yago Gonzalez-Velo answered: How long a device lasts in space depends on where it is used in the space system (whether more or less shielding is provided), and in which orbit that space system is being flown. For commercial space applications, the total dose radiation related qualification is usually done for TID levels of $150 \mathrm{krad}$ or lower. In the case of a challenging mission, such as research missions around Saturn or Jupiter, the TIDs are higher and in the range of 300 krad to $1 \mathrm{Mrad}$. That gives you an idea of the environment that designers will design towards, and the TID levels that we are testing these devices at are much higher.

(37:[50]50) Gabriela Kissling said: Have you performed any tests on the cyclability of your devices as a function of the radiation dose? Is there a difference in the lifetime of the devices or the amount of cycles you can perform before the devices break down as a function of the amount of radiation they have been exposed to?

Yago Gonzalez-Velo replied: In previous studies, we have conducted some assessment of the cyclability of chalcogenide based devices, however we did not look into the maximum number of cycles that could be obtained after exposure. Our previous work showed that the HRS/LRS ratio increases after total dose 
exposure and we also observed that the cycling lifetime was preserved or not changed after exposure to TID, i.e. we checked that the 10 kcycle operations occurring on the controls also occurred after exposure. ${ }^{1,2}$ Such results were obtained on a given manufacturing process and would probably be process dependent. We did not conduct a broader study towards an optimization of cyclability with total dose.

1 Y. Gonzalez-Velo, H. J. Barnaby, M. N. Kozicki, P. Dandamudi, A. Chandran, K. E. Holbert, M. Mitkova and M. Ailavajhala, IEEE Trans. Nucl. Sci., 2013, 60, 4563-4569.

2 J. L. Taggart, Y. Gonzalez-Velo, D. Mahalanabis, A. Mahmud, H. J. Barnaby, M. N. Kozicki, K. E. Holbert, M. Mitkova, K. Wolf, E. Deionno and A. L. White, IEEE Trans. Nucl. Sci., 2014, 61, 2985-2990.

(38:[51]51) Daniele Ielmini commented: Regarding the annealing affect, your data show that the HRS resistance decreases during the annealing at high temperature, while the LRS resistance remains approximately constant. Can you comment on the possible mechanism behind such resistance drift?

Yago Gonzalez-Velo answered: This is an effect that we are interested in understanding better and it certainly requires additional characterization, which will need different techniques. However, as a comment, I would say that based on the results that we obtained I would look at having a better understanding of the existence and impact of nanocrystallite phases, such as $\mathrm{Ag}_{2} \mathrm{Se}$, in the overall electrolyte resistance in the HRS state $\left(\mathrm{Ag}_{2} \mathrm{Se}\right.$ has different bandgap and resistivity than the base chalcogenide film).

(39:[52]52) Daniele Ielmini asked: Again about the annealing-induced resistance drift, would it be possible that Ag gets clustered within the electrolyte, thus causing enhanced percolation in the film? Do you have evidence and information abut the Ag content in the electrolyte before and after annealing?

Yago Gonzalez-Velo responded: Yes, that could be a possibility. We know that nanocrystallites form, we and other researchers in the literature have identified binary and ternary phases forming depending on the manufacturing recipe. The formation of silver cannot be dismissed, even though we have no characterization of it. Perhaps techniques such as transmission electron microscopy (TEM) could help in this case. We do not have information about the silver content before and after annealing, not at this point. It would be an interesting follow-up study.

(40:[53]53) Philip Bartlett said: I have a naïve question. Gamma rays are high energy, is there any difference in the absorption cross sections for the different materials? Are there any areas in the device where they will be absorbed more strongly, or are they just absorbed uniformly everywhere?

Yago Gonzalez-Velo responded: This is an interesting question. Gamma-rays, because they are high-energy photons, allow for dose reinforcement to be avoided at the interface between the high-Z material and low-Z material. Such effects could occur with X-rays, for example, but not with gamma-rays, which enable minimization of the effect and are in part the reason for the use of a standard for device total dose qualification. Additionally, in our case the irradiator used is 
a Gammacell 220, where the configuration of the Co-60 sources provides photons impinging from all directions on the samples, also minimizing dose nonuniformities.

(41:[54]54) Geoffrey Burr commented: Your tests involve a particular device area. Would you expect to encounter more or less radiation-induced problems for larger or smaller-size devices? For instance, if a high-energy event can significantly increase conductance, then a larger area means more chance of this happening. What trend would you expect with different device areas?

Yago Gonzalez-Velo responded: This is a question that can be interpreted in several ways: if you are thinking of a high energy event, such as the heavy-ion interaction within the ReRAM cells, in the same way that people look at radiation effects and mention transient effects, such as single event effects, this is something that we have not fully characterized. I have focused here on total dose effects. However, an interaction by itself will lead to an absorption/deposition of energy, i.e. potentially leading to total dose effects (even the heavy-ion interaction). For devices with decreasing area the probability for an interaction to occur will decrease since the target volume will be smaller. However, if nanocrystallite growth occurs (as observed in the XRD characterization presented in this work) then, because the crystallites would grow in smaller ReRAM cells, the overall effect of the crystallite on the ReRAM cell might be higher in the case of a smaller ReRAM cell. My guess would be a trade-off/balance between having small ReRAM cells providing a lower cross-section or area to be seen by impinging particles, an increased number of cells within an array, and a possible increase of the TID impact on the ReRAM due to the crystallite growth. The impact of such crystallites still needs to be investigated and characterized though, and their dimensions and composition evaluated.

(42:[55]55) Vladimir Kolosov said: Why do you not do transmission electron microscopy (TEM) in addition to X-ray diffraction? Are there some specific problems with the sample preparations for TEM in your system?

Yago Gonzalez-Velo answered: We are interested in conducting TEM characterization of our samples, it will be very interesting indeed. It is a question of scheduling the different analysis, as the duration is incremental and it ends up taking a long time to perform. This is something that we plan on doing in the near future. Concerning the preparation of the samples for TEM, there are indeed some specific problems with sample preparation. We have conducted a couple of TEM studies of samples using the same material system and the issues, such as the time spent between lamella extraction and TEM visualization, are critical. It is also important to avoid keeping the electron beam on one spot for a long time as we have already observed electron beam induced crystallization on the cross section/lamella samples in previous cases. This, as an example, can have an impact on the EDS profiling done with TEM.

(43:[56]56) Ruomeng Huang asked: I have a question regarding the material preparation process. How uniformly has the $\mathrm{Ag}$ been incorporated into the GeSe layer after photodoping? Is it homogeneously doped? In addition, what is the 
effect of the annealing process on the Ag doping profile in the GeSe layer? Is this photodoping process applicable to other metal and dielectric systems?

Yago Gonzalez-Velo responded: This is an interesting question. We do indeed conduct photodoping of the chalcogenide film with silver through UV light exposure. ${ }^{1,2}$ This enables the formation of the electrolyte through the incorporation of $\mathrm{Ag}$ inside the chalcogenide film. It has been shown in the literature that Ag exhibits a doping front in chalcogenide films that is quite constant (uniform doping with depth), ${ }^{3}$ however I would comment that this is probably not applicable in the case of our ReRAM devices where the existence of the metal/ electrolyte/metal structure might enable some electric field effect to occur due to the difference in work functions between the anode and cathode. A previous study conducted within our group showed that, using an EDS line scan on a TEM lamella, the Ag profile was not uniform throughout our films, with poor Ag doping close to the Ni cathode. ${ }^{4}$ The photodoping would be applicable to chalcogenide films of various compositions where $\mathrm{Ag}$ or $\mathrm{Cu}$ are used as the active metals. ${ }^{2,3}$

1 Y. Gonzalez-Velo, H. J. Barnaby, M. N. Kozicki, P. Dandamudi, A. Chandran, K. E. Holbert, M. Mitkova and M. Ailavajhala, IEEE Trans. Nucl. Sci., 2013, 60, 4563-4569.

2 A. Mahmud, Y. Gonzalez-Velo, H. J. Barnaby, M. N. Kozicki, M. Mitova, K. E. Holbert, M. Goryll, T. L. Alford, J. L. Taggart and W. Chen, IEEE Trans. Nucl. Sci., 2017, 64, 2292-2299.

3 M. Frumar and T. Wagner, Curr. Opin. Solid State Mater. Sci., 2003, 7, 117-126.

4 D. Mahalanabis, Y. Gonzalez-Velo, H. J. Barnaby and M. N. Kozicki, IEEE Trans. Electron Devices, 2014, 61, 3723-3730.

(56:[57]57) Ilia Valov said: These are nice results on the radiation effects in AgGeSe devices. What about the materials aspect? Is it the only material that shows this radiation resistant property? Do you have some idea which materials would be preferred?

Yago Gonzalez-Velo replied: In the last few years we have investigated the TID tolerance of systems such as $\mathrm{Ag}-\mathrm{Ge}_{40} \mathrm{~S}_{60}$ (ref. 1) and $\mathrm{Cu}-\mathrm{SiO}_{2}$ (ref. 2) and both led to $\mathrm{PMC} / \mathrm{ReRAM} / \mathrm{CBRAM}$ cells that exhibited good resistive switching after exposure to high TID. However, we have not investigated the material changes occurring in these systems yet. It is also good to mention that there are several works that have been presented in the literature ${ }^{3-6}$ not only on CBRAM but also on OxRAM that showed that such material systems also provided ReRAM cells that exhibited resistance switching at high TID. From a preference point of view, I think that there is work to be done to downselect but having a stable material with minimal crystallization would probably be my guess.

1 P. Dandamudi, H. Barnaby, M. Kozicki, Y. Gonzalez-Velo and K. Holbert, IEEE Trans. Nucl. Sci., 2013, 61, 1726-1731.

2 W. Chen, H. Barnaby, M. Kozicki, A. H. Edwards, Y. Gonzalez-Velo, R. Fang, K. Holbert, S. Yu and W. Yu, IEEE Trans. Nucl. Sci.,, 2015, 62, 2404-2411.

3 E. DeIonno, M. D. Looper, J. V. Osborn, H. Barnaby and W. M. Tong, Radiation effects studies on thin film TiO2 memristor devices, IEEE Aerospace Conference Proceedings, 2013.

4 M. J. Marinella, S. M. Dalton, P. R. Mickel, P. E. Dodd, M. R. Shaneyfelt, E. Bielejec, G. Vizkelethy and P. G. Kotula, IEEE Trans. Nucl. Sci., 2012, 59, 2987-2994.

5 L. Zhang, R. Huang, D. Gao, P. Yue, P. Tang, F. Tan, Y. Cai and Y. Wang, 2011, IEEE Trans. Electron Devices, 58, 2800-2805.

6 R. Fang, Y. Gonzalez-Velo, W. Chen, K. Holbert, M. N. Kozicki, S. Yu, Appl. Phys. Lett., 2014, 104, 183507. 
(100:[100]100) Gianluca Milano opened a general discussion of the paper by Ludovic Goux: In your work, the device performances were associated to the empty volume of the chalcogenide layers. This means that the device performances, such as the writing speed, depend on the film density and porosity. As recently reviewed by Valov et al., ${ }^{1}$ moisture can be adsorbed and incorporated in nanoporous films both with or without chemical interaction, affecting the resistive switching behaviour. Indeed, besides impacting the electronic transport properties of the films, moisture can affect the electrochemical reactions (in particular the counter electrode reaction) and can thus regulate the switching kinetics. As reported by Tsuruoka et al., ${ }^{2}$ in the case of $\mathrm{Ta}_{2} \mathrm{O}_{5}$, lower film densities result in more water molecules being incorporated in the active material that may enhance the redox reactions at the metal-electrolyte interfaces, thus influencing the resistive switching properties, such as electroforming. Are the device performances observed in your chalcogenide films with different densities related to the different spatial constriction conditions that facilitate the migration of host ions in less dense chalcogenide electrolytes or to the different amounts of adsorbed moisture from the ambient environment that affect the switching kinetics?

1 I. Valov and T. Tsuruoka, J. Phys. D, 2018, 51, 413001.

2 T. Tsuruoka, I. Valov, S. Tappertzhofen, J. V. D. Hurk, T. Hasegawa, R. Waser and M. Aono, Adv. Funct. Mater., 2015, 25, 6374-6381.

Ludovic Goux replied: That is a good point. We unfortunately have no information about whether the quantity of moisture varies with the density of our material, so it is difficult to assess whether it is the spatial condition or the moisture itself that is the origin of the properties. I would rather expect that it is the spatial conditions because moisture would fill the pores more and it would indeed reduce the switching speed but I would also expect that it would degrade retention, which is not what we observe. The question remains open still.

(101:[101]101) Ilia Valov commented: Have you performed experiments to determine the role of the short range order and also that of the density of the electrolyte materials? The particular surrounding in the cluster may also play a role in determining the performance. This could, for example, be studied using X-ray absorption experiments.

Ludovic Goux answered: This is indeed a good point. We have recently started some EXAFS experiments, however we have no results so far.

(102:[102]102) Niloufar Raeishosseini said: I have a question about the thermal stability. Considering GST as the phase change material, how do the authors confirm the phase of the material is stable during the experiment, specifically while the external voltage is applied? In Fig. 2 of the article (DOI: 10.1039/ C8FD00115D), the sample prepared for XRD measurement should be prepared on a $\mathrm{Si}$ wafer rather than $\mathrm{SiO}_{2}$ to avoid the XRD peak of the $\mathrm{SiO}_{2}$ substrate, which is almost between 20 and 25. To be more concise, a high-temperature XRD is suggested; in this case, the authors can investigate the sample by elevating the temperature in steps until a transition temperature around 250 and $280{ }^{\circ} \mathrm{C}$ is reached. It seems just annealing the samples at 250 and taking the XRD will not 
be enough to realize the stability of the samples. The MIT transition is likely to happen at $250-280{ }^{\circ} \mathrm{C}$ or even less annealing temperature depending on the samples.

Ludovic Goux responded: Thank you for the suggestion of this experiment. We agree that some parasitic local phase change of the GeTe material during switching is not excluded.

(103:[103]103) Ella Gale asked: You mention in your paper (DOI: 10.1039/ C8FD00115D) that factor $\gamma$ is a geometric factor from a random walk in 3dimensions. Why did you use a 3-D random walk? 1-, 2-, and 3-D random walks have very different properties. For example, walkers can easily get stuck in a queue in 1-D, but this is much less likely in 3-D. If this is a conducting filament with ions hopping along it, would it be better modelled as a 1-D random walk? For example, the attempt frequency would be reduced if there was any queuing effect and crucially reduced by different amounts dependent on the number of walkers (ions) moving along the filament, so this would change as the device is used. Furthermore, there has been work in this field where the filaments are modelled as not 1-, 2- or 3-D but as fractals. See the work by J. S. Lee et al. ${ }^{1}$ where the fractal dimension was found to be 2.54 , so a fractal dimension is likely the best description of the filament structure.

1 J. S. Lee, S. B. Lee, S. H. Chang, L. G. Gao, B. S. Kang, M. -J. Lee, C. J. Kim, T. W. Noh and B. Kahng, Phys. Rev. Lett., 2010, 105, 205701.

Ludovic Goux responded: This is an interesting point. We actually do not think that 1-D walk would better describe the cation motion because field lines are not uniform. On the other hand, 3-D is also an overestimate, we agree. Probably the fractal dimension would indeed be the most appropriate. Anyway, we would like to note here that the equation was introduced only for the qualitative interpretation of the motion as a hopping mechanism.

(104:[104]104) Ilia Valov commented: Under applied voltage there won't be a chance to observe a random walk. At these conditions ion velocity is exponentially dependent on the electric field. I would suppose that there will be no 3D motion.

(105:[105]105) Alexander Shluger asked: What is the driving force for $\mathrm{Cu}$ to form a compact filament? Why do $\mathrm{Cu}$ atoms get together to form this type of a cylinder? What is the evidence that $\mathrm{Cu}-\mathrm{Cu}$ interaction can assemble randomly diffusing $\mathrm{Cu}$ atoms into such structure?

Ludovic Goux answered: We performed ab initio simulations, which are unpublished, showing $\mathrm{Cu}$ that is thermodynamically more stable in clusters than as dispersed atoms. Besides, numerous works, including TEM imaging, have shown that $\mathrm{Cu}$ filaments or clusters are formed in CBRAM devices.

(106:[106]106) Alexander Shluger said: You put a lot of emphasis on free volume, which basically suggests that you think that $\mathrm{Cu}$ is moving by interstitial 
hopping. Another mechanism is more efficient in some cases, such as when an interstitial atom pushes an atom out of its site and substitutes it, and other collective mechanisms, see the studies by Frank et al. ${ }^{1}$ and F. Faupel et al. ${ }^{2}$ The so called interstitialcy mechanism is very efficient, particularly for interstitial atom diffusion. ${ }^{3}$ Why do you think that $\mathrm{Cu}$ is moving by hopping in this system? Did you consider other collective mechanisms? They will cause different free volume dependence.

1 W. Frank, A. Horner, P. Scharwaechter and H. Kronmuller, Mater. Sci. Eng., 1994, 179, 3640.

2 F. Faupel, W. Frank, M. Macht, H. Mehrer, V. Naundorf, K. Rätzke, H. R. Schober, S. K. Sharma and H. Teichler, Rev. Mod. Phys., 2003, 75, 237.

3 B. Sadiqh, T. J. Lenosky, S. K. Theiss, M. Caturla, T. D. Rubia and M. A. Foad, Phys. Rev. Lett., 1999, 83, 4341.

Ludovic Goux answered: This is an interesting remark, thank you for highlighting these previous studies. We did not have an a priori view of the cation motion mechanism, our interpretation of hopping through the empty volume just follows from our observation of faster motion in more porous matrix. However, in case the substitution scenario would be able to support our data, we would be open to considering it.

(107:[107]107) Geoffrey Burr said: I have a question about Fig. 11 in your paper (DOI: 10.1039/C8FD00115D). It's obvious that the bar chart drawing is a cartoon, but I noticed in the text that you report SET time as a function of the pulse-length that induces a SET state. However, it is possible that this SET event is influenced by the pulses that come before this final pulse, perhaps even the aggregate time exposure. This would imply that different pulse sequences or orders would end up producing a different "SET duration". I also would point out that in a memory application, it is most likely that this SET event, which we want to be fast, will come after the device has been sitting in an "unselected" state, which could mean a small negative polarity across the device. What has been your experience in terms of the dependence of the "SET time" on the time between pulses or on the polarity experienced by the device just before the SET pulse?

Ludovic Goux replied: This is a very good point indeed. For CBRAM devices, where switching is controlled by cation drift and redox, we typically observe that an ISPP write programming sequence is more efficient than a single write pulse having the same length as the last ISPP pulse, indicating that the sequence does something to the device that is not translated immediately in the electronic conduction. This can be expected from the impact on the cation drift and concentration profile. Obviously, opposite polarities will also affect this differently. This observation is also valid for OxRAM devices and I invite you to read a complete study of ours on this topic. ${ }^{1}$

1 "C.Y.Chen et al., IEDM 2016"

(138:[108]108) Dirk Wouters said: In your presentation, interestingly, you attribute the better stability of the Ge-Se vs. Ge-Te based CBRAM device to the stronger bond of $\mathrm{Cu}$ with Se than with $\mathrm{Te}$. As $\mathrm{Cu}$ has a stronger bond to Se than to Te, would that also affect the electrical conductivity of the filament, especially the 
Ludovic Goux answered: It is indeed possible that for tiny filaments, when the conductivity may no longer be described as originating from a $\mathrm{Cu}$ nanowire, the filament surface conductivity contributes. In that respect, I have no insight for the time being whether we would observe a difference between Ge-Se and Ge-Te electrolytes.

(110:[110]110) Stanley Williams opened a general discussion of the paper by Elia Ambrosi: Building on the talk earlier by Prof. Yuchao Yang about what happens to the electrodes when you apply such large voltages, such as are applied in your work, did you look at the electrodes using some type of microscopy during or after your experiments? Did you see any deformation in the electrodes when you applied these very large voltages? Were you blowing things up, blowing bubbles, etc.? Did you look for changes in the electrodes? If you don't look for them explicitly, you won't see them.

Elia Ambrosi answered: We have not performed microscopy observations in our device before or during the operation of the devices. However, we might safely rule out any bubbling effect because the operating voltage was much lower than reported in the previous work by Prof. Yuchao Yang.

(111:[111]111) Stanley Williams commented: I suggest strongly that you use an appropriate microscope and look at your devices as you're doing the switching or after the experiments are over, in the case of very small devices. We did that 10 years ago and were rather astounded when we saw craters that had erupted out of our electrodes. Significant changes can occur, as we saw earlier from Prof. Yuchao Yang's talk. The structure that you have after you've applied these potentials can be completely different from what was originally built.

Elia Ambrosi responded: We thank you for your precious advice and we will look into studying the device structure before and after device operation by appropriate microscopy techniques.

(112:[112]112) Daniele Ielmini remarked: Regarding the possibility of bubble formation in our devices, although we have not inspected our devices for possible bubbling because of the extremely small active area of $70 \mathrm{~nm}$ diameter of the bottom electrode, I would rather exclude this possibility for several reasons. First, the devices formed at relatively low current, generally $10 \mu \mathrm{A}$, thanks to the onetransistor/one-resistor (1T1R) structure with an integrated transistor. The low current forming allows the control of the local electrical parameters and Joule heating in the forming transient, thus preventing destructive thermal runaway. Second, the bubble sizes evidenced in the previous paper by Dr Yuchao Yang (DOI: 10.1039/c8fd00113h) are a few $100 \mathrm{~nm}$ at least, which makes even the smallest observed bubble much larger than the device itself. Third, our devices generally show endurance in the range of $1 \mathrm{E}^{7}$ to $1 \mathrm{E}^{8}$, which alone makes the bubbling and its related degradation extremely unlikely. 
(113:[113]113) Dirk Wouters said: In your work, you compare switching in different metal oxide layers, i.e. hafnium oxide and silicon oxide. However, another difference is that the $\mathrm{HfO}_{2}$ is stoichiometric, while the silicon oxide is substoichiometric $\left(\mathrm{SiO}_{\mathrm{x}}\right.$ and not $\left.\mathrm{SiO}_{2}\right)$. Why did you choose substoichiometric silicon oxide, what is the value of $x$, and did you examine the effect of different stoichiometries?

Elia Ambrosi responded: In our devices, silicon oxide is deposited by e-beam evaporation starting from a silicon monoxide solid target, thus $\mathrm{x}$ should be around 1. We chose sub-stoichiometric silicon oxide to increase the leakage current in the pristine state, thus reducing the forming voltage and improving the control of LRS/HRS. All our results with $\mathrm{SiO}_{2}$ instead of $\mathrm{SiO}_{\mathrm{x}}$ led to excessive forming voltages and unstable RRAM operation.

(114:[114]114) Dirk Wouters remarked: In your paper, you compare the switching characteristics of different oxides based on the DC $I-V$ characteristics. Did you also perform kinetic analysis? Is the time behavior also similar?

Elia Ambrosi responded: We have conducted limited analysis of the pulsed behavior of the $\mathrm{SiO}_{\mathrm{x}}$ and $\mathrm{HfO}_{2}$ RRAMs, from which it appears that the dynamic operation in the two materials is rather similar. For instance, we see a comparable increase of the set/reset voltages at decreasing pulse-width. A systematic analysis and comparison of the two materials is not yet completed.

(115:[115]115) Tony Kenyon said: When you deposit $\mathrm{Ti}$ on an oxide the $\mathrm{Ti}$ would usually oxidise, drawing oxygen out of the oxide and producing oxygen vacancies. This is typically used in Si oxide and Hf oxide, for example, to seed the interface with oxygen vacancies. In your case I would expect the same thing to happen, so how much of what you're seeing could be due to the Ti oxide layer rather than the silicon oxide? Could the switching be a bilayer effect?

Elia Ambrosi responded: Indeed, switching might be a two-layer effect because of the $\mathrm{O}$ gettering from the Ti top electrode. However, we observe strong differences in the forming characteristics in Fig. 2 of our paper (DOI: 10.1039/ C8FD00106E), which might indicate a central role of the bulk oxide, namely $\mathrm{HfO}_{2}$ or $\mathrm{SiO}_{\mathrm{x}}$, instead of the artificial $\mathrm{TiO}_{\mathrm{x}}$. Also, the LRS can become quite conductive at high compliance current, which suggests that the filament is metallic in both $\mathrm{SiO}_{\mathrm{x}}$ and $\mathrm{HfO}_{2}$, and that Ti (instead of $\mathrm{O}$ vacancy) might have a leading role in the formation of the conductive path.

(116:[116]116) Yang Li said: In Fig. 7 of your paper (DOI: 10.1039/ C8FD00106E), you reported the negative set behavior of the $\mathrm{HfO}_{2}$ ReRAM and no negative set behavior in the $\mathrm{SiO}_{\mathrm{x}}$ devices. In Fig. 8 of your paper, you showed the retention characterization of the devices and the $\mathrm{SiO}_{\mathrm{x}}$ ReRAM showed larger resistance fluctuation. You attribute both the negative set and the resistance retention behavior to the energy barrier height for defect migration. I am just wondering if they're kind of contradictory, since if you have a higher energy barrier for defect migration in $\mathrm{SiO}_{\mathrm{x}}$, as explained for the negative reset behavior, 
shouldn't we also expect less fluctuation for the $\mathrm{SiO}_{\mathrm{x}}$ devices in the retention experiment?

Elia Ambrosi replied: In both $\mathrm{SiO}_{\mathrm{x}}$ and $\mathrm{HfO}_{2}$, the HRS shows resistance fluctuations, although both oxide RRAMs show good data retention with no data loss even after $1 \mathrm{~h}$ annealing at $260^{\circ} \mathrm{C}$. Also note that the retention characteristics of the $\mathrm{SiO}_{\mathrm{x}}$ and $\mathrm{HfO}_{2}$ RRAMs should be compared at equal resistance level, which is not possible for some relatively high resistance value in $\mathrm{SiO}_{\mathrm{x}}$ which we could not attain with a $\mathrm{HfO}_{2}$ RRAM. Overall, the retention characteristics of the two RRAM devices look rather similar. Regarding the energy barrier for the negative set and retention, one should note that the two processes are rather different, therefore the relevant energy barrier might also have different values. For the negative set, the defects are activated by the application of a negative voltage at the top electrode. On the other hand, the data loss mechanism in the retention experiment is the spontaneous diffusion (no voltage applied) in the conductive path. In conclusion, given the different physical mechanisms involved in the negative set and retention experiment, the different behavior of the two RRAM devices in these experiments does not look like a contradiction.

(117:[117]117) Alexander Shluger commented: You state that the breakdown voltage of $\mathrm{SiO}_{\mathrm{x}}$ is $20 \mathrm{MVcm}^{-1}$, which is much higher than the well-established breakdown voltage for $\mathrm{SiO}_{2}$ films of about $10 \mathrm{MVcm}^{-1}$, see for example work by Verweij and Klootwijk. ${ }^{1}$ How do you explain why the $\mathrm{SiO}_{\mathrm{x}}$ in your system is much more stable with respect to dielectric breakdown than $\mathrm{SiO}_{2}$ films?

\section{J. F. Verweij and J.H. Klootwijk, Microelectron. J., 1996, 27, 611-622.}

Elia Ambrosi replied: There are at least two important differences between standard $\mathrm{SiO}_{2}$ and the $\mathrm{SiO}_{\mathrm{x}}$ dielectric used in our RRAM devices. First, the device is $\mathrm{SiO}_{\mathrm{x}}$ with $\mathrm{x}$ equal to about 1 . Second, the $\mathrm{SiO}_{\mathrm{x}}$ layer has already been subject to breakdown during the forming operation, as seen in Fig. 2 of our paper (DOI: 10.1039/C8FD00106E). As a result, the breakdown event taking place during the negative set voltage can be viewed as a second breakdown, where the local conductive path is further degraded as a result of the application of a negative voltage. This is similar, for instance, to the hard breakdown event, compared to the soft breakdown event that occurred at forming.

(118:[118]118) Alexander Shluger asked: So this is not a real dielectric breakdown?

Elia Ambrosi replied: Indeed, we might view our estimated breakdown field of $20 \mathrm{MVcm}^{-1}$ as an effective breakdown field for the onset of negative set, not to be compared to the known breakdown field of $\mathrm{SiO}_{2}$.

(130:[119]119) Vikas Rana enquired: How much current compliance has been used during the retention measurement?

Elia Ambrosi responded: Before the retention experiment, multiple ReRAM devices were initially prepared in various resistance states. Different HRS and LRS 
values were obtained by changing the reset stop voltage $\mathrm{V}_{\text {stop }}$ and the compliance current $\mathrm{I}_{\mathrm{C}}$, respectively. The LRS was initially programmed in a range from about $10 \mathrm{k} \Omega$ to $100 \mathrm{k} \Omega$, corresponding to programming compliance currents between $100 \mu \mathrm{A}$ and $10 \mu \mathrm{A}$, respectively. During the retention experiment, after each annealing step, the resistance of each device was read using a $-0.2 \mathrm{~V}$ reading voltage at the top electrode, while keeping the series transistor biased at relatively large gate voltage to minimize the voltage drop across the transistor. So no compliance was used during the retention experiment, as the devices were read with a voltage much lower than the set/reset voltage.

(131:[120]120) Vikas Rana remarked: And what is the impact of the current compliance on your retention? What is the device size used in the retention test?

Elia Ambrosi responded: In Fig. 8 of our paper (DOI: 10.1039/C8FD00106E), both the $\mathrm{HfO}_{2}$ and $\mathrm{SiO}_{\mathrm{x}}$ devices show slightly larger resistance fluctuations for a large initial LRS $(\mathrm{R} \approx 100 \mathrm{k} \Omega$ ), namely for the resistance states obtained with a low programming compliance current $\mathrm{I}_{\mathrm{C}}$. However, both oxides show good retention with no data loss. The device size is about $70 \mathrm{~nm} \times 70 \mathrm{~nm}$.

(132:[121]121) Jonas Deuermeier asked: You're concluding that the forming process is very different, whereas the $I-V$ curves are very similar, although you're using different hafnium oxide thicknesses. The forming comparison was done looking at the $5 \mathrm{~nm}$ hafnium oxide, seen in Fig. 2 of your paper (DOI: 10.1039/ C8FD00106E), whereas the $I-V$ comparison refers to the $10 \mathrm{~nm}$ hafnium oxide, e.g. Fig. 4 of your paper. It seems that the characteristics in general become similar by simply using a hafnium oxide twice as thick as the silicon oxide. In order to check this hypothesis, I was wondering if you tested the negative breakdown of the $10 \mathrm{~nm}$ hafnium oxide up to $-10 \mathrm{~V}$, as you do for the silicon oxide.

Elia Ambrosi replied: In this work, we have not systematically tested the negative breakdown in $10 \mathrm{~nm} \mathrm{HfO}_{2}$, although the negative set voltage markedly increased with respect to the $5 \mathrm{~nm}$ thick $\mathrm{HfO}_{2}$, as we could increase the stop voltage to a range (e.g., $-4 \mathrm{~V}$ ) which was not accessible with the $5 \mathrm{~nm}$ thick $\mathrm{HfO}_{2}$. See Fig. 5c and d of our paper (DOI:10.1039/C8FD00106E).

(133:[122]122) Jonas Deuermeier said: Is the $10 \mathrm{~nm}$ hafnium oxide formed at $4 \mathrm{~V}$ or at $8 \mathrm{~V}$ ?

Elia Ambrosi replied: Forming of the $10 \mathrm{~nm} \mathrm{HfO}_{2}$ occurred at about $8 \mathrm{~V}$.

(134:[123]123) Sherif Ibrahim said: Regarding the observed negative set during the device cycling, did you manage to eliminate that negative set and get a proper reset of the device?

Elia Ambrosi replied: In general, we believe that the negative set can be explained by the breakdown of the bottom electrode interface with the dielectric layer, therefore the negative set can be attenuated or solved only by either increasing the characteristic breakdown field $\mathrm{F}_{\mathrm{BD}}$ or by reducing the electric field 
during reset below the critical threshold. In this work, we manage to eliminate the negative set in the $\mathrm{HfO}_{2}$ device by increasing the $\mathrm{HfO}_{2}$ thickness, hence reducing the field below $\mathrm{F}_{\mathrm{BD}}$. Another solution, also implemented in our work, is the adoption of relatively low $\mathrm{V}_{\text {stop }}$ values to reduce the operating electric field. On the other hand, we believe that the key approach to radically solve the negative set is to engineer the bottom electrode interface to increase $\mathrm{F}_{\mathrm{BD}}$. We are currently conducting some experiments to optimize the bottom electrode interface to improve $\mathrm{F}_{\mathrm{BD}}$ in the $\mathrm{HfO}_{2}$.

(136:[124]124) Carlo Ricciardi enquired: Why did you choose carbon as the bottom electrode? If the $\mathrm{Ti}$ ions are migrating, did you check for any Ti-C compound at the interface?

Elia Ambrosi answered: In general, it is good practice to adopt a rather inert bottom electrode for RRAM, as opposed to a rather oxidizable metal for the top electrode. This enables asymmetric structure and a polarity dependent operation of the device with positive-voltage set and negative voltage reset, with the voltages applied to the top electrode. Previous studies have also pointed out that the inert bottom electrode increases the immunity to the negative set. ${ }^{1}$ Given the relevance of the negative set for improving the RRAM endurance, an inert electrode will thus strengthen the device against endurance failure. ${ }^{2}$

Carbon is known to be an inert material with low reactivity especially at high temperature. Our previous work also suggested that RRAM devices with a C bottom electrode were immune from negative set and showed a high endurance. ${ }^{3}$ For these reasons, we adopted $\mathrm{C}$ as the bottom electrode in our RRAM. We did not check for the presence of $\mathrm{Ti}$ at the bottom electrode or for possible reaction of $\mathrm{Ti}$ with C. This is indeed possible, although metal carbides, such as TiC, are also known to be rather inert compounds that react with oxide only at relatively high temperature. Therefore, the reaction of $\mathrm{Ti}$ with $\mathrm{C}$ at the bottom electrode should not be critical for the negative set.

1 C. Y. Chen, L. Goux, A. Fantini, A. Redolfi, S. Clima, R. Degraeve, Y. Y. Chen, G. Groeseneken and M. Jurczak, IEEE International Electron Devices Meeting, Institute of Electrical and Electronics Engineers, San Francisco, 2014.

2 S. Balatti, S. Ambrogio, Z. Wang, S. Sills, A. Calderoni and N. Ramaswamy, IEEE Trans. Electron Devices, 2015, 62, 3365-3372.

3 A. Bricalli, E. Ambrosi, M. Laudato, M. Maestro, R. Rodriguez and D. Ielmini, IEEE Trans. Electron Devices, 2018, 65, 122-128.

(137:[125]125) Stefano Brivio said: You suggest that the operation of all the investigated devices relies on Ti drift and diffusion. The conduction mechanisms of the different devices in the various resistance states may reveal differences or additional similarities among the investigated systems. In this respect, have you analyzed and compared the conduction mechanisms of such devices in the various resistance states?

Elia Ambrosi responded: To test the conduction mechanism, we characterized the $I-V$ curves, which allow the assessment of the voltage dependence of resistance. In general, we found quasi-linear current, i.e. constant conductivity, for the LRS and a strongly non-linear transport in the HRS. The LRS showed an increased 
non-linearity at increasing resistance or equivalently for lower compliance current of the set transition. In general, a linear increase of current with voltage can be attributed to a metallic conduction in the conductive path, whereas the non-linear behavior can be attributed to Poole-Frenkel conduction at the defects, such as metallic clusters, oxygen vacancies, or grain boundaries. More detailed temperature-dependent studies are currently being conducted to confirm this picture.

(140:[126]126) Ilia Valov said: About carbon, I think, there is an additional effect. It is supporting the way you suppress the oxygen reaction. We have seen this in our previous papers. ${ }^{1,2}$. In addition, the mobility of $\mathrm{Ti}$ in $\mathrm{HfO}_{2}$ has been documented by the group of G. Bersucker ${ }^{3}$ and in a paper by Privitera et al. ${ }^{4}$

1 Nature Nanotechnlogy 2016.

2Adv Mater 2015.

3 G. Bersucker et. al.

4 Microelectronic engineering 2013.

Elia Ambrosi answered: We agree that C might prevent oxidation at the bottom electrode, hence contributing to the good performance under reset and the endurance of our RRAM devices.

(802:[127]127) Asal Kiazadeh communicated: Thanks for the work you have presented during the Faraday Discussion. Regarding Fig. 2 in your paper (DOI:10.1039/C8FD00106E), in the electroforming step of the pristine states of different devices, the compliance current (CC) alters almost two orders of magnitude. Does this mean that CC is not fixed through the gate of the series transistor for a certain level? In addition, it doesn't look like the reason for the changing CC is due to different leakage at the pristine state. Some with higher leakages reach lower CC values. Following the previous question, when the CC is higher, the required voltage is higher too, then how we can interpret the distribution of the forming voltages in a proper way? One needs to consider different sizes of filament during electroforming, right?

Elia Ambrosi communicated in reply: The reason for the different compliance currents in Fig. 2 of our paper ((DOI: 10.1039/C8FD00106E) is that various values of the gate voltage $\mathrm{V}_{\mathrm{G}}$ were used for different devices. The compliance current is indeed controlled by the gate voltage, however $\mathrm{V}_{\mathrm{G}}$ was deliberately changed, thus resulting in various compliance currents. Neither the compliance current was influenced by the initial leakage of the device, nor was the forming voltage influenced by the compliance current, which plays a role only to control the final resistance of the LRS after forming.

(803:[128]128) Sweety Deswal communicated: What are the conduction and switching mechanisms in the two $\mathrm{HfO}_{\mathrm{x}}$ and $\mathrm{SiO}_{\mathrm{x}}$ devices? How are these mechanisms contributing for better performance of the $\mathrm{SiO}_{\mathrm{x}}$ device? Or what are the reasons for the better performance of the $\mathrm{SiO}_{\mathrm{x}}$ device? 
Elia Ambrosi communicated in reply: The conduction mechanism is believed to be drift in the metallic Ti filament for the LRS and Poole-Frenkel in the HRS. The switching mechanism is most probably Ti migration induced by the electric field. The improved performance of the $\mathrm{SiO}_{\mathrm{x}}$ is because of the larger breakdown field for the negative set event, which enables a larger resistance window and a higher endurance.

(119:[129]129) Ella Gale opened the discussion of the paper by Masa-aki Haga: You stated that you had a $\mathrm{pKa}$ gradient that was generated and used as the driving force for proton movement. That is exciting. Did you design that at the start? How closely did it follow your design? And, if so, what are the design rules? If I wanted an arbitrary $\mathrm{pKa}$ gradient, how would I go about designing it?

Masa-aki Haga answered: We have published two papers concerning the energy storage based on proton-coupled electron transfer (PCET) reactions. ${ }^{1,2}$ Based on the basic studies for the PCET reaction for the $\mathrm{Ru}(\mathrm{II})$ and $\mathrm{Ru}$ (III) states, we can expect a pKa change caused by the redox reaction. In the previous study, we reported a proton-rocking-chair-type redox capacitor with the same combination of two RuNH-OH and RuCH-OH multilayer films on ITO electrodes sandwiched with neutral $\mathrm{NaClO}_{4}$ aqueous solution. In this capacitor, under the galvanostatic condition, the stable charge-discharging process took place, see ref. 36 in our paper (DOI: 10.1039/C8FD00098K), and during the charging process the electric energy was stored as a $\mathrm{pH}$ gradient between the two electrodes. For the discharging, the electric energy was released as a $\mathrm{pH}$ neutralization process. The guiding principle for the design of a protonic memristor arose from the idea of replacing the neutral aqueous solution in the previous redox capacitor with a proton-conducting polymer, such as $\mathrm{P} 4 \mathrm{VP}$, resulting in the charge storage memory device. The design role is to arrange the molecules with different $\mathrm{pKa}$ values each other. A better design for the system is that the molecules are hydrogen-bonded and arranged within the assembly. A list of the pKa values for the organic compounds is available and on the other hand you can find the $\mathrm{pKa}$ data set for the inorganic complexes, which is still limited. The proton pump in the biological membrane is the most studied system, so you will have a chance to get an idea for the new artificial system.

1 D. Motoyama, K. Yoshikawa, H. Ozawa, M. Tadokoro and M. Haga, Inorg. Chem., 2017, 56, 6419-6428.

2 K. Yoshikawa, D. Motoyama, Y. Hiruma, H. Ozawa, S. Nagano and M. Haga, ACS Appl. Mater. Interfaces, 2018, 10, 26990-27000.

(120:[130]130) Ella Gale remarked: Is that why you used Ru? I presume that it would be different if you chose different ions?

Masa-aki Haga replied: The redox chemistry of the ruthenium (II/III) complexes has been well known as a small reorganization energy system and the oxidation states of both $\mathrm{Ru}(\mathrm{II})$ and $\mathrm{Ru}(\mathrm{III})$ are substitutionally inert. Thanks to pioneers such as Nobel laureate Prof. H. Taube, Prof. T. J. Meyer, and Prof. R. W. Murray and their contribution in this field, we can easily design the functional $\mathrm{Ru}$ complexes by choosing the auxiliary ligands around the $\mathrm{Ru}$ ion. Therefore, we chose $\mathrm{Ru}$ complexes. 
(121:[131]131) Stanley Williams said: About 20 years ago, I was working in what I thought was molecular electronics. It took me about 6 years to realise I wasn't doing molecular electronics at all. What I thought was molecular electronics was actually switching in the electrodes, i.e. the electrical contacts to the molecules. We wrote a paper in 2004 in which we looked at different types of molecules. ${ }^{1}$ Interestingly, the $I-V$ characteristics of the molecules all looked the same. In fact, see the curves in our previous work (Fig. 1), ${ }^{1}$ which look identical to the $I-V$ curve in Fig. 8 of your paper (DOI: 10.1039/C8FD00098K). What type of control experiments have you done? How convinced are you that what you see in your devices is molecular related? Your system is very complex. Why should it give the same $I-V$ curve that we saw 14 years ago with very different molecules. I described the background for these observations previously. ${ }^{2}$

1 D. R. Stewart, D. A. A. Ohlberg, P. A. Beck, Y. Chen, R. S. Williams, J. O. Jeppesen, K. A. Nielsen and J. Fraser Stoddart, Nano Lett., 2004, 4, 133-136.

2 R. S. Williams, IEEE Spectrum, 2008, 45, 28-35.

Masa-aki Haga responded: Thank you for your critical comments about the similarity between the $I-V$ hysteresis loop of your Pt/organic monolayer/Ti devices and our present device. We have been following your work from the beginning of our molectronics study, but I could not realize the similarity to the $I-V$ plots in your previous work (Fig. 1) ${ }^{1}$ until you commented on our data. The device structures are totally different from each other. Concerning the two terminal electrodes in the devices, in your case $\mathrm{Pt}$ on $\mathrm{a} \mathrm{SiO}_{2} / \mathrm{Si}$ wafer and Ti electrodes were used, but both electrodes were ITO electrodes for our measurement. Further, the molecules for your device were monolayer films of $\sim 2-3 \mathrm{~nm}$, whereas in our device the Ru multilayer + sandwiched polymer films were $100 \mathrm{~nm}$ thick. Therefore, the conducting mechanism is totally different. Furthermore, our "complex" molecules were tethered through the chemical bonding between the phosphonate groups of the molecule and ITO electrode, which has been a well-known method to anchor the molecules onto the metal oxide surfaces ${ }^{.2,3}$
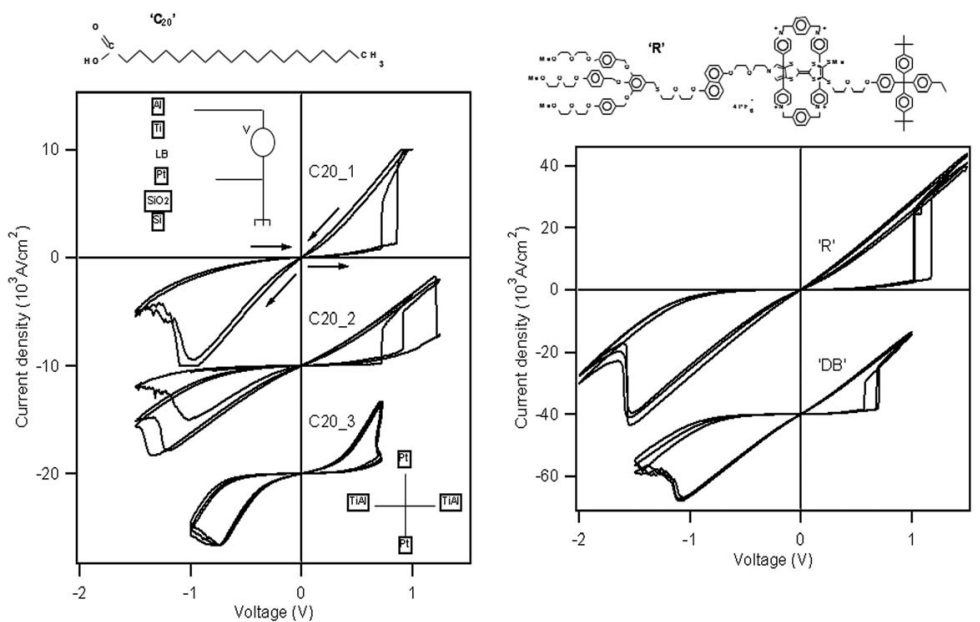

Fig. 1 Molecule independent electrical switching curves reproduced from Stewart et al. ${ }^{1}$ 
We have been studying the surface electrochemistry of the redox-active molecules immobilized on an ITO surface, and these studies have been extended into layer-by-layer (LbL) multilayer films. By the judicious selection of redox potentials in the molecules, photo responsive hetero LbL films through ET rectification have been achieved, ${ }^{4}$ furthermore interesting humidity-controlled rectification switching in single molecular junctions for our Ru complexes has been recently found. ${ }^{5}$. Through this study, the redox-active Ru LbL multilayer films are stable under the applied potential and thermally robust up to $200^{\circ} \mathrm{C}$. By changing the Ru complexes from $\mathrm{RuNH}-\mathrm{OH}$ and $\mathrm{RuCH}-\mathrm{OH}$ to the complex bearing N-methyl protected groups, RuNMe-OH and RuCMe-OH, see Fig. 2 in our paper (DOI: 10.1039/C8FD00098K), the $I-V$ plots did not show little current flow, which indicated our devices are sensitive to the immobilized molecular structures. At this stage, we could not have any answer why the $I-V$ plots in your work and ours showed similar hysteresis loop at almost the same voltages, even the film thickness is so different from each other. From the preliminary analysis of the $I-V$ plots for our devices, the space-chargelimited conduction model is fitted for the low-to-high current transition. The energy barriers at the molecule/electrode interface through the hydrogen bonding between the RuNH-moieties and P4VP drastically changed under the voltage application and the charge accumulated at the interface by the $\mathrm{pKa}$ gradient.

1 D. R. Stewart, D. A. A. Ohlberg, P. A. Beck, Y. Chen, R. S. Williams, J. O. Jeppesen, K. A. Nielsen and J. Fraser Stoddart, Nano Lett., 2004, 4, 133-136.

2 G. Cao, H. G. Hong and T. E. Mallow, Acc. Chem. Rev., 1992, 25, 420-427.

3 E. L. Hanson, J. Guo, N. Koch, J. Schwartz and S. L. Bernasek, J. Am. Chem. Soc., 2005, 127, 10058.

4 T. Nagashima, H. Ozawa, T. Suzuki, T. Nakabayashi, K. Kanaizuka and M. Haga, Chem. Eur. J., 2016, 22, 1658-1667.

5 H. Atesci, S. J. van der Molen et al., Nature Nanotech., 2018. 13, 117-1218.

(122:[132]132) Stanley Williams said: You have to be really careful in terms of the control experiments. We were using rotaxanes, for which a Nobel prize was awarded. We felt we were onto something. I worked on this for 6 years before I figured out we'd been fooling ourselves.

Masa-aki Haga responded: The surface redox-active coordination network system has a potential to pursue various functions not only for electronics but also photochemical and catalytic properties, therefore we will continue to study the surface molecular devices.

(123:[133]133) Rainer Waser said: We have shown, for two presumably molecular switching systems, both rose bengal and $\mathrm{Cu}$ TCNQ, that we could leave out the molecules completely and switch them in the same way. I've seen from your $I-V$ curves that your currents go up to $4 \mathrm{~mA}$. What kind of switching do you believe you have?

Masa-aki Haga responded: We have missed the literature survey for your rose bengal and $\mathrm{Cu}$ TCNQ systems. We should know these. If possible, please let me know these papers. Our device area is $0.95 \mathrm{~cm}^{2}$, which is relatively large. Therefore, the current density reached is $\sim 4-10 \mathrm{mAcm}^{-2}$. We have reported a protonrocking-chair-type redox capacitor with the same combination of two RuNH-OH and $\mathrm{RuCH}-\mathrm{OH}$ multilayer films on ITO electrodes sandwiched with neutral 
$\mathrm{NaClO}_{4}$ aqueous solution. In this capacitor, under the galvanostatic condition, the stable charge-discharging process took place ${ }^{1}$ and, during the charging process, the electric energy was stored as a $\mathrm{pH}$ gradient between two electrodes. For the discharging, the electric energy was released as a $\mathrm{pH}$ neutralization process. The guiding principle for the design of a protonic memristor arose from the idea of replacing the neutral aqueous solution in the previous redox capacitor with a proton-conducting polymer, such as $\mathrm{P} 4 \mathrm{VP}$, resulting in the charge storage memory device. I propose the redox-induced switching of $\mathrm{RuNH}-\mathrm{OH}$ and $\mathrm{RuCH}-$ $\mathrm{OH}$ on the acid-base pair hydrogen bonding site, as shown in Fig. 6 of our paper (DOI: 10.1039/C8FD00098K), induced the charge accumulation (proton or negative charge) at the hetero layer junction according to the $\mathrm{pKa}$ difference, becoming a stronger acid for one side and a on the other side a stronger base, which is the driving force for the high conducting state. This stayed until the reverse redox reaction occurred by the reverse potential bias. This process might be relevant to the ATP synthesis by the $\mathrm{pH}$ gradient in the biomembrane.

1 K. Yoshikawa, D. Motoyama, Y. Hiruma, H. Ozawa, S. Nagano and M. Haga, ACS Appl. Mater. Interfaces, 2018, 10, 26990-27000.

(125:[134]134) Philip Bartlett said: To continue on that point, I think it would be better for this plot seen in Fig. 8 of your paper (DOI: 10.1039/C8FD00098K) to show the current density rather than the current. In your paper you focus on cations, the two Ru complexes on the two electrodes are both cationic and you focus on the movement of the protons. What are the compensating anions involved here? In both layers of the Ru complexes on the two electrodes you have some corresponding anions to maintain electroneutrality, and in the protonated poly(vinylpyridine) there will be some associated anions. Is it the same anion in the electrode layers and in the poly(vinylpyridine)? Have you looked at changing the anion? What effect does it have on the properties of the system?

Masa-aki Haga answered: Thank you for your comment. We have changed the $I-$ $V$ plots by converting the y axis to the current density from the current value. Regarding the cation/anion movement on the LbL films, the RuNH-OH and RuCH$\mathrm{OH}$ complexes showed proton-coupled electron transfer (PCET) reactions on the ITO electrode. We have measured the electrochemical quartz crystal microbalance (EQCM) measurements for the Ru complexes having PCET reaction immobilized on the ITO EQCM chip in aqueous solution. Upon the oxidation of RuNH-OH from the $\mathrm{Ru}(\mathrm{II})$ to $\mathrm{Ru}(\mathrm{III})$ state, the charge imbalance was cancelled out by the proton release/ capture on the redox reactions of the protonated $\mathrm{Ru}(\mathrm{II})-\mathrm{NH} /$ deprotonated $\mathrm{Ru}(\mathrm{III})-\mathrm{N}$. On the other hand, RuNMe-OH showed large frequency change for the charge compensation by the captured fo anions. Under the solid state films, the ITO|(RuNH-OH $)_{n}$ electrodes were hydrogen bonded with P4VP, therefore little anion movement may take place on the ITO|(RuNH-OH $)_{n}$ layer, but in the P4VP polymer layer the proton movement is induced by the hydrogen-bonded water molecules, so the hydroxide anions play an important role. The study on the cation/anion effects for the $I-V$ plots will be planned, in addition to the effect of deuterium water.

(126:[135]135) Philip Bartlett asked: If you look at Fig. 9 in your paper (DOI: $10.1039 / \mathrm{C} 8 \mathrm{FD} 00098 \mathrm{~K}$ ) this describes a kind of battery in which protons move 
from the redox film on one electrode, through the electrolyte, to the redox film on the other electrode. You could make the same thing, for example with a lithium ion battery, replacing the mobile protons with $\mathrm{Li}^{+}$.

Masa-aki Haga replied: Thank you for your comment. In our previous paper, ${ }^{1}$ we reported a proton-rocking-chair-type redox capacitor composed of ITO|(RuNH$\mathrm{OH})_{n}$ /aqueous neutral $\mathrm{NaClO}_{4} /(\mathrm{RuCH}-\mathrm{OH})_{\mathrm{n}} \mid \mathrm{ITO}$ as two sandwiched electrode devices. By modifying the $\mathrm{NH}$ group to the other Li ion trapping one, a new type of Li ion battery will be realized.

1 K. Yoshikawa, D. Motoyama, Y. Hiruma, H. Ozawa, S. Nagano and M. Haga, ACS Appl. Mater. Interfaces, 2018, 10, 26990-27000.

(127:[136]136) Niloufar Raeishosseini said: Because humidity is the main factor in the proton conduction mechanism, I wonder if the authors measured the device under different conditions or not? The authors mentioned "the strong dependence of the $I-V$ plots on the humidity and acid treatment of the P4VP film indicates that the protonated state of P4VP plays an important role for proton conduction," therefore, it is expected that they show the relevant graphs and electrical measurements by discussing the effect of humidity. The authors also mentioned that "in order to elucidate the humidity effect on RuNH-OH/P4VP and $\mathrm{RuCH}-\mathrm{OH} / \mathrm{P} 4 \mathrm{VP}$, the proton conductivity was measured by changing the humidity RH values." However, it is not clear which graph is related to changing the RH values? In Fig. 5 of the paper (DOI: 10.1039/C8FD00098K), it is suggested to determine the oxidation and reduction peaks of the cyclic voltammetry measurement and discuss the electrochemical property to attract the readers' attention. In Fig. 8 and 10 of the paper, the cycles of the $I-V$ sweeps are shown. How many cycles does the device endure? Under which condition is the device measured? Are all of the electrical measurements under a humid condition? In Fig. 9 of the paper, is the measurement done under the humid condition and, if so, what is the percentage of humidity? Did the authors compare the differences in the $I-V$ curves for the humid condition and non-humid environment?

Masa-aki Haga responded: Thank you for your important question. I have not discussed details about the relative humidity effects on our P4VP devices in this paper due to still on-going experiments with my collaborator, Prof. Yuki Nagao at JAIST. With his permission, I have showed the preliminary results for the relative humidity (RH) dependence of the proton conductivities of P4VP with different layer-structured films, such as sapphire|P4VP, sapphire|(RuNH-OH $)_{3} / \mathrm{P} 4 \mathrm{VP}$, and sapphire|(RuNH-OH $)_{3} / \mathrm{P} 4 \mathrm{VP}-\mathrm{HCl}$ for the protonated state of $\mathrm{P} 4 \mathrm{VP}$ by the mixing of P4VP polymer with hydrochloric acid before spin-coating (Fig. 2), in which the proton conductivities increased dramatically upon the protonation on P4VP. Furthermore, the EIS measurements of the ITO $\mid(\mathrm{RuNH}-\mathrm{OH})_{3} / \mathrm{P} 4 \mathrm{VP} /(\mathrm{RuCH}-\mathrm{OH})_{3-}$ IITO devices have been performed under different RH conditions (Fig. 3). When the relative humidity ( $\mathrm{RH}$ ) increased from $40 \%$ to $90 \%$, the semicircle on the EIS spectra became smaller, indicating the resistivity became smaller, thus the conductivity increased. We are still continuing the $\mathrm{RH}$ effect on the conductivity, which will be published in a subsequent paper, including the reproducibility. All the $I-V$ measurements in the paper have been carried out at the ambient 


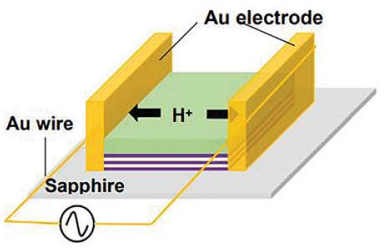

- P4VP

- P4VP-HCl

- $\mathrm{Ru}-\mathrm{CH}+\mathrm{P} 4 \mathrm{VP}$

- $\mathrm{Ru}-\mathrm{CH}+\mathrm{P} 4 \mathrm{VP}-\mathrm{HCl}$

- Ru-NH+P4VP

- Ru-NH+P4VP-HCl

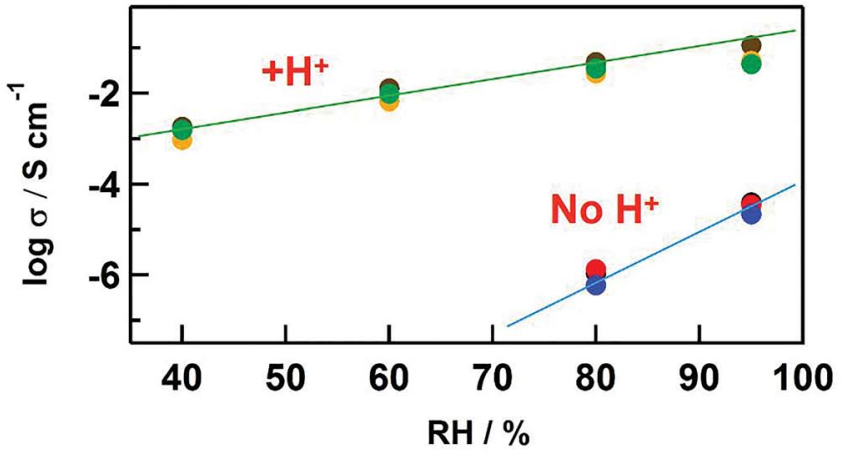

Fig. 2 Proton conductivities of P4VP with different layer-structured films on sapphire under different relative humidity $(\mathrm{RH})$ environments. Protonated state of P4VP was prepared by the mixing of P4VP polymer methanol solution with hydrochloric acid.

condition at room temperature ( $\mathrm{RH} \sim 40 \%)$. The $I-V$ sweeps were retained for about 40 cycles, after the cycles the hysteresis loops on the I-V curves collapsed, leading to the non-linear $I-V$ curves. When the water vapor was blown to the

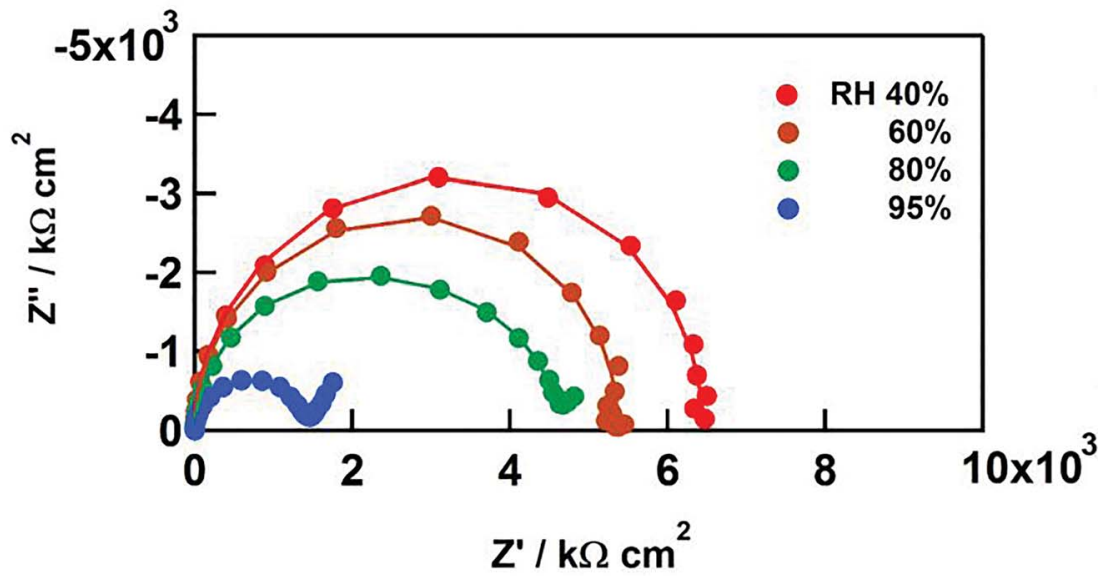

Fig. 3 Humidity $(\mathrm{RH})$ dependence of the Nyquist plots for the ITO|(RuNH-OH) $3 / \mathrm{P} 4 \mathrm{VP} /$ $(\mathrm{RuCH}-\mathrm{OH})_{3} \mid \mathrm{ITO}$ device. 
device, the increase of the current was observed. We need more quantitative $I-V$ measurements under the controlled RH conditions in the near future.

(128:[137]137) Sanne Berg asked: You mentioned that you could maintain 50 cycles for your devices, I was wondering if this is the stability limit and if breakdown of the device is observed after this point. If that is the case would you have suggestions on how to improve the stability and lifetime of your devices?

Masa-aki Haga answered: Regarding the device repeatability, the hysteresis loop became smaller on the $I-V$ plots after 50 cycles and finally only nonlinear $I-V$ curve were obtained in the present devices. The present two-terminal device has not been sealed, so the device performance will be improved by the use of the sealant after clarifying the optimum humidity condition. In addition, the thickness of the sandwiched P4VP may be affected by the humidity and the protonation, which might influence the device stability.

(129:[138]138) Daniele Ielmini said: Is forming necessary to initiate switching in these devices or are they forming free?

Masa-aki Haga answered: No forming was applied for the fabrication. Two modified ITO electrodes were combined by pressing.

(135:[139]139) Stanley Williams remarked: You made a very interesting comment about the fact that your devices didn't require forming; neither did ours. It turns out that was a red herring. The molecules were actually reacting with the metals in the system. This actually gave us a great clue as to how to make forming free devices with oxides. You have to do some very intense control experiments with these molecular systems. Just the fact you have forming-free operation doesn't mean much. Chemical reactions between the molecules and electrodes may be important.

Masa-aki Haga responded: Chemical reactions or physisorption between the molecules and electrode surface induced the energy level alignment and the workfunction change took place. ${ }^{1}$ In particular, the surface modification of the ITO surface with phosphoric acid has been reviewed in the literature. ${ }^{2}$ Therefore, the combination of the molecules bearing phosphoric acid with an ITO electrode is suitable for the forming-free devices for organic electronics.

1 H. Ishii, K. Sugiyama, E. Ito and K. Seki, Adv. Mater., 1999, 11, 605-625.

2 S. A. Paniagua, A. J. Giordano, O. K. Smith, S. Barlow, H. Li, N. R. Armstrong, J. E. Pemberton, J. L. Brédas, D. Ginger and S. R. Marder, Chem. Rev., 2016, 116, 7117-7158.

(139:[140]140) Monica Santamaria said: Fig. 10 of your paper (DOI: 10.1039/ C8FD00098K) shows an impedance spectrum in the Nyquist representation (Fig. 10a) and the equivalent circuit you used to simulate the behaviour of the device (Fig. 10b). In the experimental section some details on how the spectra were recorded are missing. You stated in the experimental section that you employed an a.c. signal amplitude of $50 \mathrm{mV}$. This is usually a sinusoidal signal superimposed to a continuous signal. I have not referred to a reference as this was written by the 
authors. Which was the latter? It is important to establish if such potential was lower or higher with respect to the switching potential. Further comments, the $\mathrm{X}$ and $\mathrm{Y}$ axes are in $\mathrm{k} \Omega$ not in $\mathrm{k} \Omega \mathrm{cm}^{2}$. Could you please normalize for the surface the real and imaginary components of the impedance in the spectrum? The magnitude of the employed constant phase elements and the corresponding best fitting exponents are not reported in the caption, therefore it is not possible to evaluate how physically reasonable the employed model is. Also, the reported spectrum looks like a depressed semicircle, thus it seems that only one time constant is enough to fit it. The authors should provide a zoom to show if other smaller semicircles are present.

Masa-aki Haga replied: For the impedance measurement, we employed the normal EIS measurement with a sinusoidal signal with an amplitude of $50 \mathrm{mV}$. The description in our paper (DOI: 10.1039/C8FD00098K) made you confused, so in the proof corrections we will delete several words; i.e. with respect to the reference. According to your comment, we will use a new y axis of $\mathrm{k} \Omega \mathrm{cm}^{2}$ for Fig. 10 in our paper. Also, we will add the constant phase elements (CPE) fitting parameter in the caption of Fig. 10 of our paper. Also, I have provided the magnified version of the EIS spectra for your reference (Fig. 4).

a

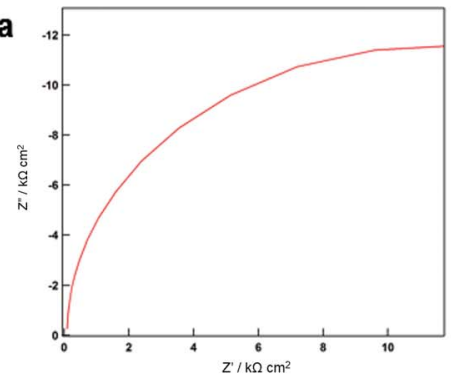

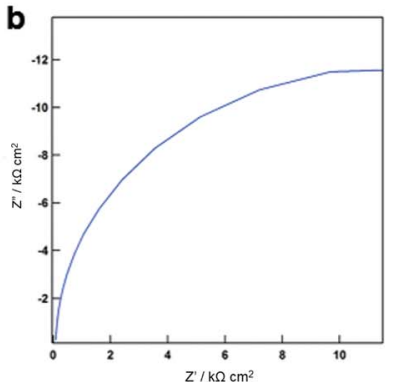

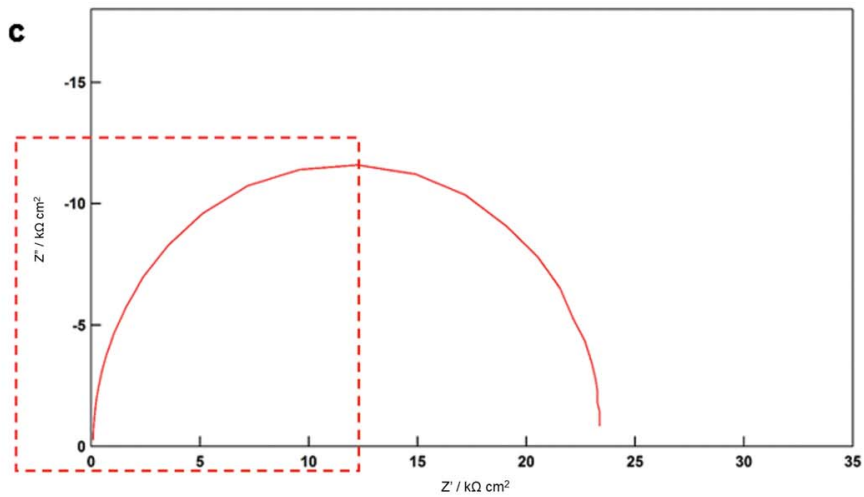

Fig. 4 (a) Magnified raw data for the EIS spectra, (b) fitted data for the EIS spectra, and (c) raw data for the ESI spectra. 
(142:[141]141) Ella Gale said: I can clearly see the bioinspiration in your work; however, what would a protonic coordination network or memristor offer technologically?

Masa-aki Haga responded: Although the present study was just a proof-ofconcept for protonic molecular devices, my vision for the protonic coordination network or memristor devices is to fabricate large-area sensor devices at the environment-human interface to act as alternatives to the human five senses.

(143:[142]142) Sayani Majumdar said: I have a question about the switching time scales of the proton conducting devices. Have you measured the device switching response at different time scales? How fast can the device switch? In the humid environment, does the switching timescale change? A good control experiment to see if there is an effect of the molecule on the switching would be to look for the effect of the humidity on the switching times. Because, if there is no effect of the molecules, then humidity wouldn't affect the switching mechanisms.

Masa-aki Haga replied: Thank you for your question about the switching time. Our device is based on the surface molecular modified electrochemistry on ITO, so the switching time was controlled by the heterogeneous electron transfer rate between the Ru complexes and ITO electrode and the electron hopping within the LbL multilayer film. ${ }^{1}$ Since the present device was composed of the molecular film and polymer films in the solid state, the response time became slower, however the switching timescale falls within a range of $\sim 1 \mathrm{~ms}$ for the positive bias and range of $\sim 1 \mathrm{~s}$ for the negative bias. I have shown the preliminary data for the current response under the pulse voltage (Fig. 5). We have not measured the

\section{Current on/off response upon the successive potential pulses}

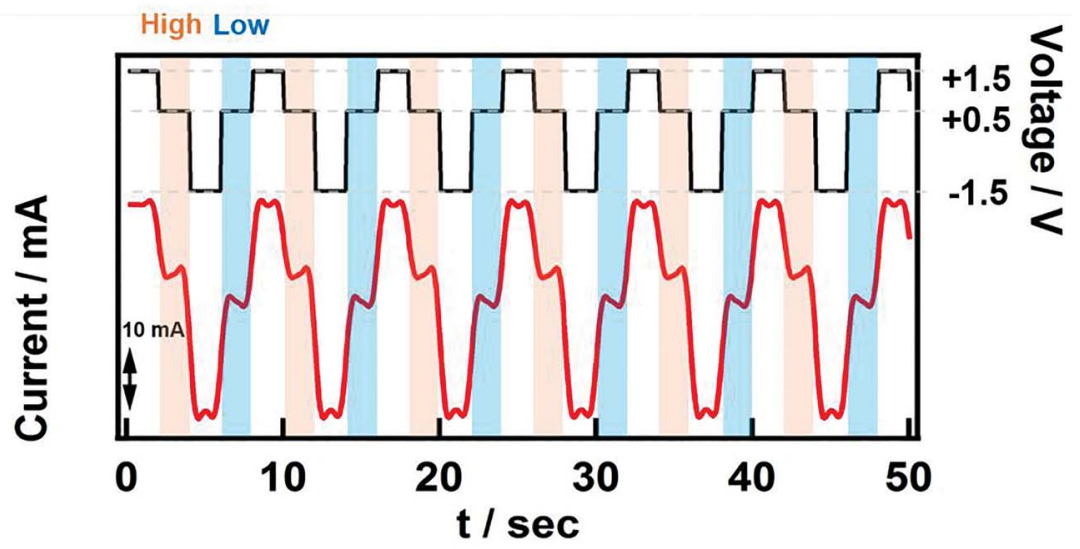

Fig. 5 Current on/off response upon the successive potential pulses. 
switching time response upon the change of humidity, but further experiments are planned in the near future.

1 T. Nagashima, T. Suzuki, H. Ozawa, T. Nakabayashi, M. Oyama, T. Ishida and M. Haga, Electrochim. Acta., 2016, 204, 235-244.

\section{Conflicts of interest}

There are no conflicts to declare. 